\title{
Investigation of Factors that Determine the Performance of Wireless Sensor Systems for Large-scale on Demand Deployment
}

\author{
Tajudeen O Olasupo* ${ }^{*}$ Kehinde O Olasupo \\ Electrical and Computer Engineering, Florida Institute of Technology, 150 W University Blvd, United States
}

Copyright $@ 2017$ by authors, all rights reserved. Authors agree that this article remains permanently open access under the terms of the Creative Commons Attribution License 4.0 International License

\begin{abstract}
Most of the studies done so far consider just one or two factors that determine network behavior during experiment or analysis and reach a conclusion with that. Thereby making wireless sensor system deployment design to incur variability and non-conformance to target requirements, and increase in development time and cost. This study proposes an approach to improve deployment plan for wireless sensor network by considering the most appropriate factors that could contribute significantly to deployment performance. The study specifies some factors and demonstrates how they influence network performance using factorial coding. It also provides demonstration of an optimization approach of implementing the factors-"EASY algorithm - Eliminate, Adjust, Simulate and Yield result” on experimental measurements. This research uses factorial design, polynomial analysis, and hypothesis testing for an experiment to investigate the factors that could determine the performance of the system. Result shows that inclusion of more significant factors in a design model will enhance the model by $26 \%$. Using this approach during pre-deployment design and pre-knowledge of performance factor will help in improving the quality of service and achieve desired aim of system. It will ensure that reliable accurate models are being used in network design, planning, and implementations for improved performance.
\end{abstract}

Keywords Factorial Design, Propagation, Performance, Simulation, Wireless Sensor Network

\section{Introduction}

Wireless sensor network (WSN) is gaining more importance as the technology gets more sophisticated in the world of Internet of Things (IoT) and in large-scale WSN deployment. The opportunity for deployment of more advanced WSN creates the need to meet some network performance metrics. The performance metrics could be connectivity, coverage, reliability, durability, lifetime of network, overall quality of service, security, maintainability, and scalability. The purpose of WSN deployment is to optimally do what is required of it. WSN designers or users cannot afford to have frequent network failure or vulnerability to attack due to its roles in military, medical, and science. The demand for wireless systems is on the rising edge [1-4]. In an effort for network designer to meet these demands, and provide wireless network coverage for users, various factors which are responsible for the performance of the network need to be investigated. Some of the factors include: channel frequency, node transmission power, routing protocols, power source or type, distance among nodes. Other factors are node's antenna height and orientation, height of nodes, node architecture, life of node, human-made structures, natural terrain in various environments, and their conditions. Some of the factors are listed in literature, but insufficient study has demonstrated how the factors influence network performance Akyildiz et al. [5,6]. Hence, it makes the development and design planning to be inefficient and thereby not meeting the desired network performance. These factors and how they influence network performance need to be put into consideration during designing, planning, and deployment of sensor systems. This study presents some of these factors, how to implement them, and how to use the proposed approach when network designers or users wish to implement other factors.

Majority of the research studies done so far consider just one or two factors which could determine network behavior during experiment or analysis. Such studies design and reach a conclusion with one or two factors. Most of the end results may not optimally describe or model the scenarios [7-14].

The model used in studying the wireless sensor system process is given in Fig.1. The process has some influential factors (controllable)- $x_{1}, x_{2}, \ldots \ldots, x_{n}$ and (uncontrollable)$z_{1}, z_{2}, \ldots ., z_{m}$ on a given input that will determine the output response, $y$ of the process. The goal is to determine: the factors that are most influential on the $y$, where to set the 
influential factors in order to reduce variability in $y$; make $y$ close to the desired nominal value; and make the effect of uncontrollable factors to be minimized or blocked using a given input.

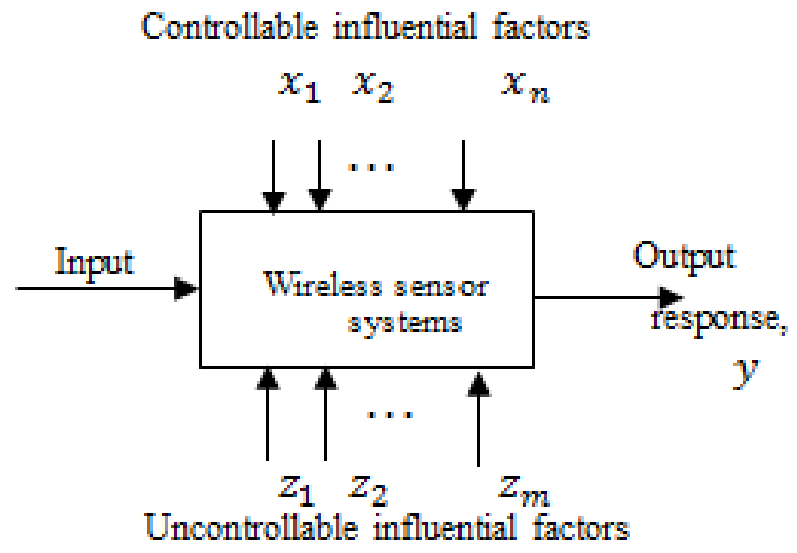

Figure.1. Model of the process or experiment

The proposed systems or simulation models in literature do not include analysis to know the significant factors to work with. Moreover, sufficient work has not been demonstrated to show the effects of these various factors on WSN performance.

Furthermore, deployment methodology - (stochastic and deterministic) is another vital challenge. In deterministic deployment, it is easy to use one or two factors for deployment design. The deployment process can be repeated with more factors if the desired performance is not attained. Conversely, it is impractical to assume this process for a stochastic large-scale deployment and harsh environment where repeat of experiment or deployment is very expensive and impossible. Also, complexity of the environment in term of large-scale, terrain variations, and landscape formation makes the whole process time consuming, costly, error prone, practically challenging, and cumbersome. For this reason, it will be imprudent and uneconomical to just design or deploy WSN without investigating the impact and the contributions of various interacting and non-interacting factors which may determine the performance of large-scale WSN deployment.

If the problem of not using more than one significant factor for the deployment design is solved, there will be positive effect on deployment decision. Subsequently, it will improve the current and future applications of WSN technology such as in military operations, post disaster recovery response, environmental quality, surveillance and border security. It is practical that there are inevitable restrictions that will not permit the considerations of all factors. However, it will be better to consider the best economical approach probably by using blocking factors approach or other analysis [15].

This research investigates how various factors affect the performance of WSN in term of connectivity and how to make judicious decision. This study performs experiments and uses factorial deign analysis for the investigation. Also, the study uses simulation algorithm to stimulate large-sale deployment. Results from the experimental analysis are compared with the simulation approach, and they show a similar outcome. The simulation and the factorial design are efficient enough to show the performance metrics of node connectivity, coverage, and scalability for WSN deployment.

The rest of the paper is organized as follows: Section II gives summary of the related work that proves the uniqueness and efficacy of this research. Section III describes the experimental campaign, the modeling, simulation approach and demonstration. The full analysis and result details are given in section IV while the section V contains the conclusion and future study.

\section{Related Work}

\subsection{Literature Review}

[16] consider electromagnetic interferences and other disturbances in industrial environment. The analysis of performance is done using entropy based approach. However, interactions among the factors are not considered. Chipara et al. [17] perform modeling and prediction of radio coverage for sensor network in indoor environment. They study the effect of distance among nodes, antenna orientation, and walls impacts. They aim to know the best model for their radio mapping tool. This work appears to leave out the important factor in the experiment. Conversely, from experimental and analytical design, this proposed study discovers that node height and transmission power affect signal propagation significantly. Also, $[18,19]$ consider just two factors which are antenna height and distance in their experiments. They do not consider the interaction effect of the factors to know which one has a significant contribution before applying it in their experiments. Therefore, to conclude that the radio signal strength indication (RSSI) variation in their experiment is modeled by fourth order polynomial might not be the optimal conclusion.

In the same vein, Temel et al. [20] present deterministic sensor deployment method based on wavelet transform (WT) is proposed. They aim to maximize coverage with minimum number of sensors. Likewise, La et al. [21] state the applications of sensor networks in monitoring the environment. They design a controller through a real-time feedback to identify the new locations of mobile sensors. In Tsai et al. [22], the authors propose fusion methods to improve crowdsourcing enhanced system sensing coverage and the quality of its decisions. Factor effect analysis are not involved in these studies.

Ohshima et al. [23] present the effect of node distance and environmental conditions on throughput as performance metric for WSN in outdoor field environment. In Ongwei et al. [24], the authors consider the effect of antenna height and human body in their experiment using RSSI level as performance criteria. Hara et al. [25] discuss the effect of 
power decay factor and distance on RSSI as the performance metric. Huang et al. [26] consider the effect of simulation of hardware design platform using RF-ZigBee design structure and PCB board. Also, Wadhwa et al. [27], investigate the impact of distance and antenna orientation on the performance of WSN to develop a protocol for energy conservation. Yaakob et al. [28] perform a simulation study on the effect of varying packet size in various bit error rate conditions on the performance of the network. NS-2 simulator is used in the analysis.

Furthermore, Otero et al. [29] carry out study that is similar to the proposed study presented in this tis report. The authors use Response Surface Methodology (RSM) and Desirability Functions to study the effect of number of nodes, sensor range and radio range. However, they do not analyze why and how significant factors would be implemented. The effects of these factors are considered to measure the four performance criteria (connectivity, coverage, cost, and transmission power) in their analysis. Analysis of variance (ANOVA) is used to simulate connectivity, coverage, and transmission model, without any experimental analysis. The authors do not demonstrate the interacting effects of factors that affect connectivity and coverage for stochastic deployment in WSN. They assume uniform distribution deployment for simulation which is not always the case in practice especially for large-scale deployment. Contrarily, in the proposed approach, non-uniform and random distribution deployment in the experimental and simulation analysis is assumed.

Likewise, in Otero et al. [30], the effects of factors affecting connectivity and coverage for stochastic deployment for WSN is not demonstrated. The authors use ANOVA, VVT, CMT, AHP to design decision making tool during developments. Based on the search on literature, it is important to test the performance of deployment using various factors. This paper shows the effects of significant factors and suggest decision making in non-uniform deployment approach for large-scale WSN based on the factors considered.

\section{Methodology and Experimental Design}

\subsection{Factorial Experimental Design and Two Way ANOVA}

\subsubsection{Factorial Experimental}

Factorial design analysis is used in an experiment to study the effects of two or more factors. For instance, if there are $a$ levels of factor $A$ and $b$ levels of factor $B$ in an experiment to yield an output at $n$ replicate, each replicate contains all $a b$ treatment combinations. This implies $2^{2}$ factorial design analysis as shown in Figure 2. The effect of factor is defined to be the change in the output response produced by a change in the level of the factor. This is frequently called a main effect because it refers to the primary factors of the interest in the experiment [15]. In the experiment using Figures 2 and 3 if factor $A$ is increased from low $\left(\bar{y}_{A^{-}}\right)$to high $\left(\bar{y}_{A^{+}}\right)$while the considering the effect of factor $B$, the effect of such change in $A$ on the output response is:

$$
\begin{aligned}
A= & \bar{y}_{A^{+}}-\bar{y}_{A^{-}}=\frac{a b+a}{2 n}-\frac{b+(1)}{2 n} \\
= & \frac{1}{2 n}[a b+a-b-(1)]
\end{aligned}
$$

That is $-1 \rightarrow-(1),+1 \rightarrow+a,-1 \rightarrow-b,+1 \rightarrow+a b$.

Similarly, if $B$ is considered, the effect of change in $B$ on the output response is:

$$
\begin{gathered}
B=\bar{y}_{B^{+}}-\bar{y}_{B^{-}}=\frac{a b+b}{2 n}-\frac{a+(1)}{2 n} \\
=\frac{1}{2 n}[a b+b-a-(1)]
\end{gathered}
$$

Also, the interaction effect of both $A$ and $B$ on the output response is:

$$
\begin{aligned}
A B & =\frac{a b+(1)}{2 n}-\frac{a+b}{2 n} \\
& =\frac{1}{2 n}[(1)-a-b+a b]
\end{aligned}
$$

where $2=2^{k-1}=2^{2-1}, k$ is the number of factor

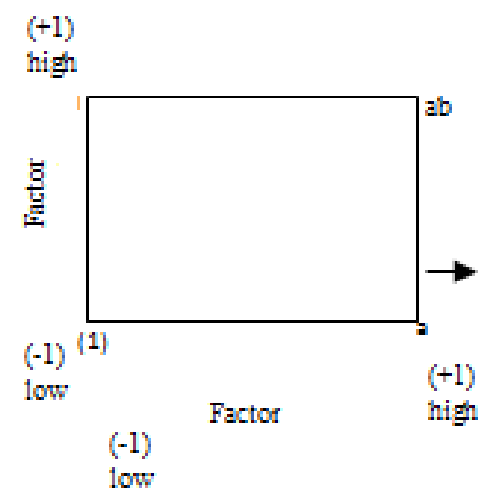

(a)

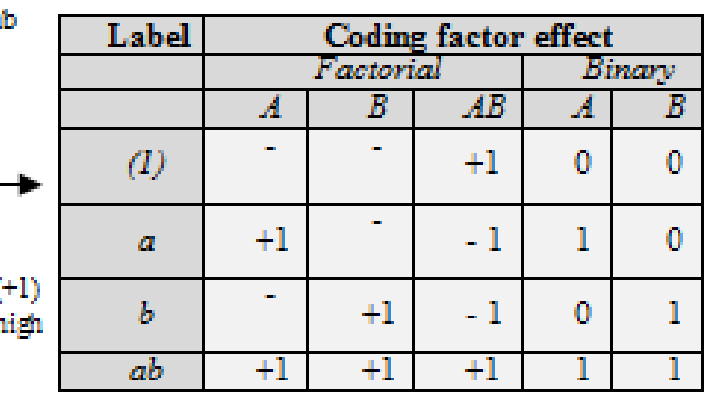

(b)

Figure. 2. (a). A two-factor factorial experiment ( $2^{2}$ factorial design) (b) factor coding to map the output response labels shown at the corners to factor levels, low (-1) and (+1) 


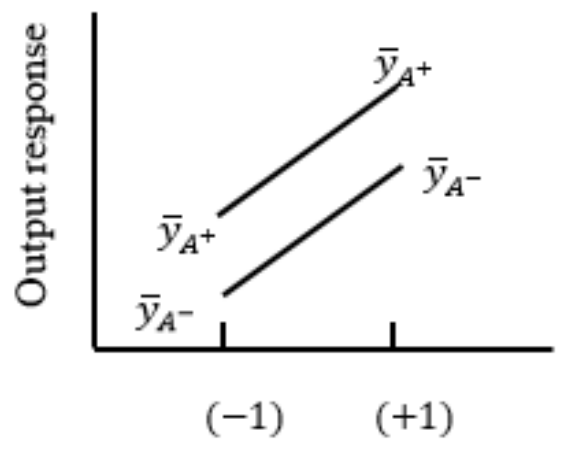

Factor B

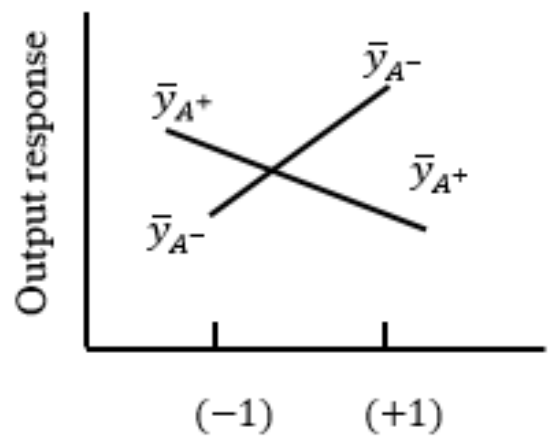

Factor B

Figure. 3. (a). Main effect-Factorial design without interaction, (b). Interaction effect-Main effect-Factorial design with interaction

From Figure 3, if the main effects A or B is positives, it indicates that there is increase from low to high and will positively impact response variable while if the effect is negative, it means factor increases from low to high and is decreasing response variable. The interacting effect $A B$ shows if there is interaction between the factors. This effect can be positive or negative. In Figure. 3b, the line cross, that means there is interacting effect.

Table 1. Algebraic Coding Signs for Calculating the Factorial Effects in the $2^{4}$ Design

\begin{tabular}{|c|c|c|c|c|c|c|c|c|c|c|c|c|c|c|c|c|}
\hline Label & \multicolumn{16}{|c|}{ Factorial effects } \\
\hline $\begin{array}{c}\text { Treatment } \\
\text { combinatio } \\
n\end{array}$ & $I$ & $A$ & $B$ & $A B$ & $C$ & $A C$ & $B C$ & $A B C$ & $D$ & $A D$ & $B D$ & $A B D$ & $C D$ & $A C D$ & $B C D$ & $A B C D$ \\
\hline (1) & +1 & -1 & -1 & +1 & -1 & +1 & +1 & -1 & -1 & +1 & -1 & -1 & +1 & -1 & -1 & +1 \\
\hline$a$ & +1 & +1 & -1 & -1 & -1 & -1 & +1 & +1 & -1 & -1 & +1 & +1 & +1 & +1 & +1 & -1 \\
\hline$b$ & +1 & -1 & +1 & -1 & -1 & +1 & -1 & +1 & -1 & +1 & +1 & +1 & +1 & -1 & +1 & -1 \\
\hline$C$ & +1 & -1 & -1 & +1 & +1 & -1 & -1 & -1 & -1 & +1 & -1 & -1 & -1 & +1 & +1 & -1 \\
\hline$a c$ & +1 & +1 & -1 & -1 & +1 & +1 & -1 & -1 & -1 & -1 & +1 & +1 & -1 & -1 & +1 & +1 \\
\hline$b c$ & +1 & -1 & +1 & -1 & +1 & -1 & +1 & -1 & -1 & +1 & +1 & +1 & -1 & -1 & +1 & -1 \\
\hline$a b c$ & +1 & +1 & +1 & +1 & +1 & +1 & +1 & +1 & -1 & -1 & -1 & -1 & -1 & -1 & -1 & -1 \\
\hline$d$ & +1 & -1 & -1 & +1 & -1 & +1 & +1 & -1 & +1 & -1 & +1 & +1 & -1 & +1 & +1 & -1 \\
\hline ad & +1 & +1 & -1 & -1 & -1 & -1 & +1 & +1 & +1 & +1 & -1 & -1 & -1 & -1 & +1 & +1 \\
\hline$a b d$ & +1 & +1 & +1 & +1 & -1 & -1 & -1 & -1 & +1 & +1 & +1 & +1 & -1 & -1 & -1 & -1 \\
\hline$c d$ & +1 & -1 & -1 & +1 & +1 & -1 & -1 & +1 & +1 & -1 & +1 & +1 & +1 & -1 & -1 & +1 \\
\hline acd & +1 & +1 & -1 & -1 & +1 & +1 & -1 & -1 & +1 & +1 & -1 & -1 & +1 & +1 & -1 & -1 \\
\hline$b c d$ & +1 & -1 & +1 & -1 & +1 & -1 & +1 & -1 & +1 & -1 & -1 & -1 & +1 & -1 & +1 & -1 \\
\hline abcd & +1 & +1 & +1 & +1 & +1 & +1 & +1 & +1 & +1 & +1 & +1 & +1 & +1 & +1 & +1 & +1 \\
\hline
\end{tabular}

Therefore, for a $2^{4}$ factorial design analysis that is used in this study, the factor coding to map the output response (y) at a labelled value to factor levels, low (-1) and high $(+1)$ is given in Table 1 . All $2^{\mathrm{k}}$ factorial designs are orthogonal designs where $k$ is the number of factor. The interaction value or product effect is the multiplication of the algebraic signs. The standard order of coding for the effects of factors in $2^{4}$ factorial design analysis for examples main effect factor, $A$ and interaction, $A B C D$ are:

$$
A=\frac{1}{n 2^{k-1}}[-(1)+a-b+a b-c+a c-b c+a b c-d+a d-b d+a b d-c d+a c d-b c d+a b c d]
$$

where $=4$, therefore,

$$
A=\frac{1}{8 n}[-(1)+a-b+a b-c+a c-b c+a b c-d+a d-b d+a b d-c d+a c d-b c d+a b c d]
$$

Similarly,

$$
A B C D=\frac{1}{8 n}[(1)-a-b+a b-c+a c+b c-a b c-d+a d+b d-a b d+c d-a c d-b c d+a b c d]
$$

In this study, the values (as shown in Figure 4) for the effects are represented in plots to describe the significance of the effects on the process. 


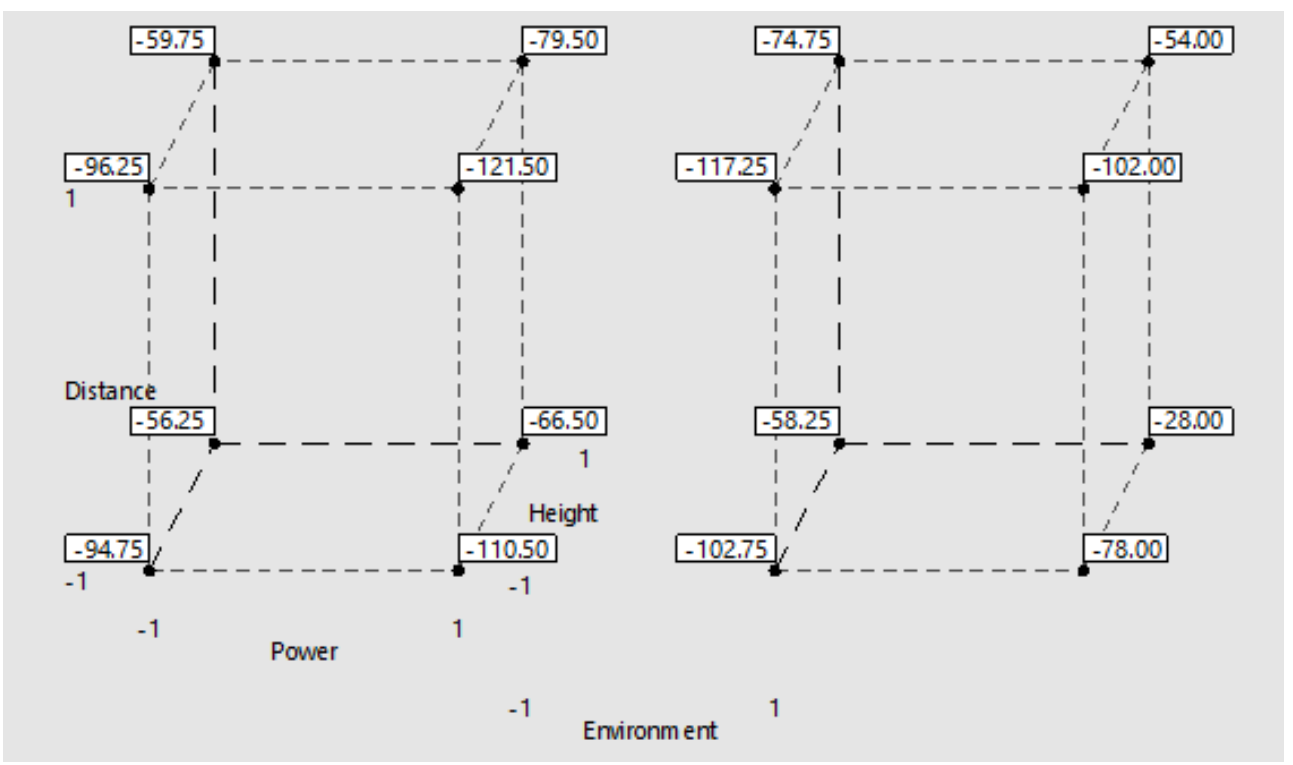

Figure. 4. An example of four-factor factorial experiment ( $2^{4}$ factorial design) representation obtained from experiment.

\subsubsection{Two Way ANOVA}

Two way analysis of variance is used to do hypothesis testing and discover interaction effect of factors in factorial design analysis by considering the P-values and F-statistic, $F_{0}$ in the analysis. Statistical parameters such as the P-value, $R^{2}$, and $R^{2}$-adjusted are obtained from two way ANOVA [31] to analyze the proposed hypothesis. In this study, the null hypothesis is that it is better to use most significant factors in a design. $R^{2}$ is the amount of total variability explained by the model as given in (7). P-value is used to know the significance of the model.

$$
R^{2}=\frac{\text { sum of square of model }\left(S S_{\text {Model }}\right)}{\text { Sum of square total }\left(S S_{\text {Total }}\right)}
$$

Also, F-statistic, $F_{0}$ computed is compared with standard table value to determine the significant of factors and models.

$$
F_{0}=\frac{S S_{T o t a l /(d-1)}}{S S_{E /(N-d)}}
$$

Similarly, adjusted $R^{2}$ takes into account the size of the model (number of factors). It decreases if nonsignificant terms are added to a model [15,31], Kupper et al. [32].

Also,

$$
R_{A d j}^{2}=1-\frac{S S_{E} / d f_{E}}{S S_{\text {Total }} / d f_{\text {Total }}}
$$

where $S S_{E}$ is error sum of squares, $S S_{\text {Total }}$ is total sum of squares and $d f$ is the degrees of freedom for observation.

\subsection{Practical Factorial Experiments}

The received signal strength indication (RSSI) is used as the performance metric or the output response in the experimental process when varying some controllable factors on a sensor node as the input. This study performs experiments in two different environments. The experiment is conducted indoor of engineering building and on flat low grass on campus with line of sight. Nodes are deployed at different distances from the server along the aisle of the building.

Each node as shown in Figure.5 is made up of a controller-Arduino platform, XBee radio-ZigBee technology with transmit power $3 \mathrm{~dB}$ operating at $2.4 \mathrm{GHz}$ band [33], XBee explorer, and 9v 3000mAH battery source. The Arduino experimental board is configured and programmed using $\mathrm{C}++$ through Arduino IDE. The XBee radio is configured and setup using the $\mathrm{X}$-CTU software. For the purpose of the first experiment, a simple point to point wireless connection is considered. One node acts as the sink node and the other as the end device or information source. The end node is moved around at different distances, heights, and transmission power. Four factors are considered in this experiment, which are distance, transmission power, height of the node, and environment type. For the factorial experiment design, each factor is set to two levels - high $(+1)$ and low (-1) in order to form a $2^{4}$ factorial design analysis. Distance is the physical separation between the nodes. Two levels of distance- $d$ used are $50 \mathrm{~m}$ (low) and $100 \mathrm{~m}$ (high). Two levels of transmission power used are $\mathrm{P} 1=-4 \mathrm{dBm}$ (low) and $\mathrm{P} 4=-2 \mathrm{dBm}$ (high). The transmission power is the amount of energy radiated from the server to the receiver. Height of the node which is the distance of the node from the ground is set to two levels: 0 feet - low and 4 feet - high. Finally, the environment types used in the factorial design are indoor (low) and flat low grass (high). RSSI values from the nodes are measured considering these factors 
simultaneously in factorial design experiment. The experiment is repeated and the values of measured performance metric are shown in Table 2. For each experimental run, the four factors are considered simultaneously and varying each factor effect as high $(+1)$ and low (-1). Factorial design analysis is used in an experiment to study the effects of two or more factors in a design. The suitable approach to dealing with several factors is to conduct a factorial analysis experiment. This is an experimental strategy in which factors are varied together, instead of one at a time. The theoretical details of factorial design are discussed by [15]. The results of the factorial design experiment are discussed in section IV of this paper.
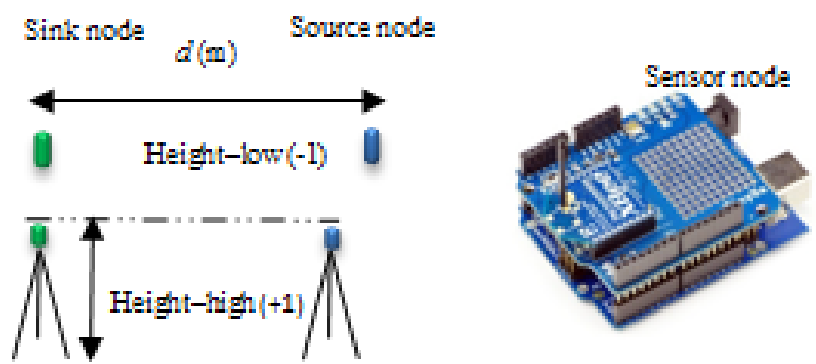

Figure 5. Point to point communication of the sensor system and a sensor node

Table 2. Sample of RSSI Readings in $\mathrm{dBm}$

\begin{tabular}{|c|c|c|c|c|c|c|c|c|c|c|}
\hline \multicolumn{9}{|c|}{ Factorial experiment design coding } & \multicolumn{5}{c|}{ Experimental run response values at each label (dBm) } \\
\hline Power & Distance & Height & Environment & Label & RSSI 1 & RSSI 2 & RSSI 3 & RSSI 4 & RSSI 5 & RSSI 6 \\
\hline-1 & -1 & -1 & -1 & $(1)$ & -71 & -72 & -72 & -73 & -71 & -67 \\
\hline+1 & -1 & -1 & -1 & a & -60 & -61 & -61 & -61 & -108 & -120 \\
\hline-1 & +1 & -1 & -1 & b & -79 & -78 & -79 & -79 & -102 & -100 \\
\hline+1 & +1 & -1 & -1 & ab & -65 & -65 & -64 & -65 & -120 & -103 \\
\hline-1 & -1 & +1 & -1 & c & -50 & -52 & -48 & -49 & -61 & -69 \\
\hline+1 & -1 & +1 & -1 & ac & -53 & -54 & -53 & -54 & -58 & -69 \\
\hline-1 & +1 & +1 & -1 & bc & -58 & -58 & -58 & -58 & -48 & -50 \\
\hline+1 & +1 & +1 & -1 & abc & -70 & -71 & -73 & -72 & -78 & -71 \\
\hline-1 & -1 & -1 & +1 & d & -90 & -87 & -90 & -91 & -116 & -112 \\
\hline+1 & -1 & -1 & +1 & ad & -92 & -91 & -93 & -92 & -79 & -71 \\
\hline-1 & +1 & -1 & +1 & bd & -95 & -93 & -95 & -95 & -102 & -108 \\
\hline+1 & +1 & -1 & +1 & abd & -90 & -93 & -91 & -92 & -106 & -99 \\
\hline-1 & -1 & +1 & +1 & cd & -59 & -60 & -61 & -59 & -41 & -52 \\
\hline+1 & -1 & +1 & +1 & acd & -62 & -60 & -62 & -61 & -23 & -24 \\
\hline-1 & +1 & +1 & +1 & bcd & -63 & -66 & -63 & -64 & -88 & -81 \\
\hline+1 & +1 & +1 & +1 & abcd & -67 & -66 & -67 & -66 & -61 & -48 \\
\hline
\end{tabular}

Analysis of Variance

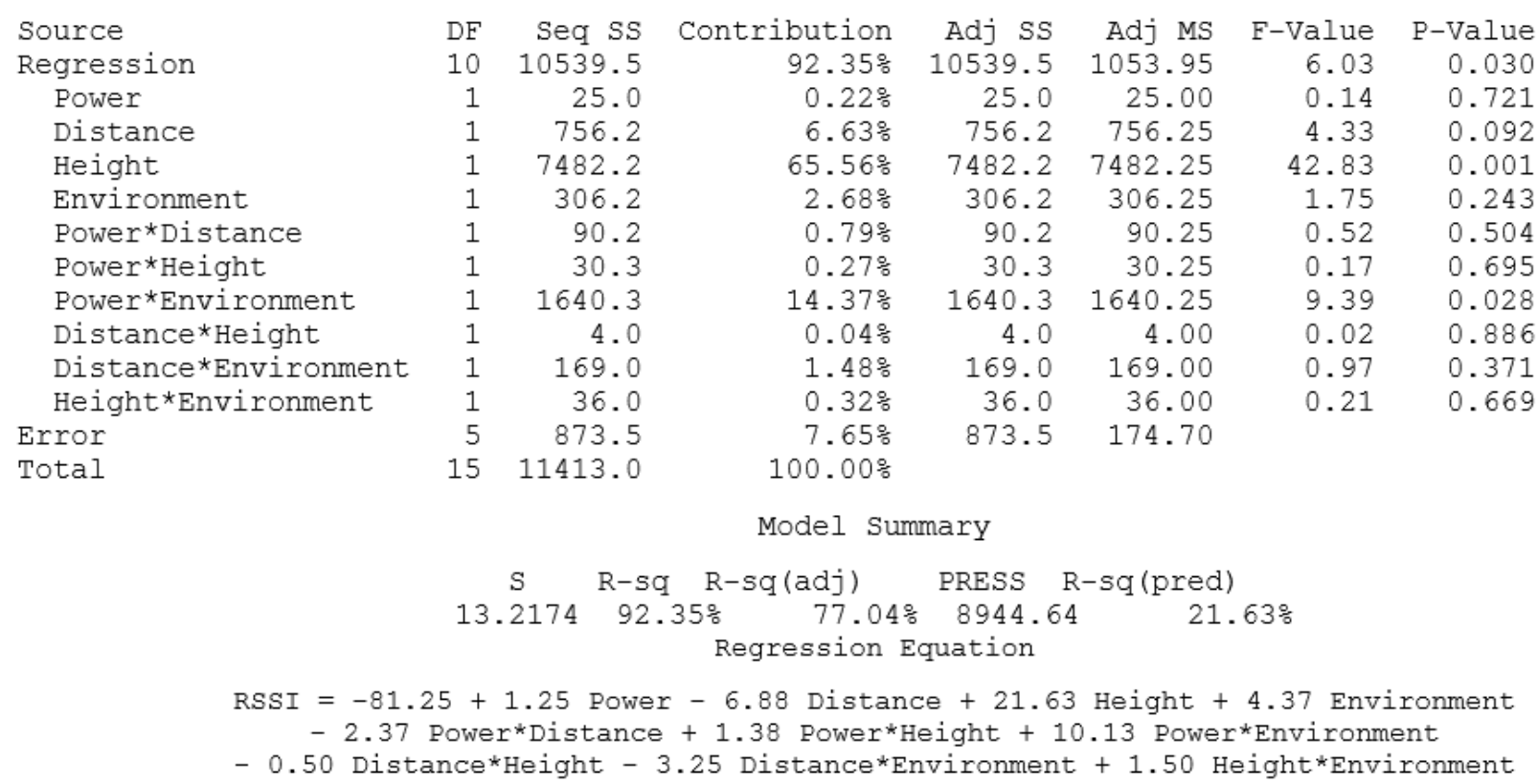

Figure 6. Two way ANOVA and polynomial model output 


\section{Results and Discussion}

The results of factorial experiments are discussed here. This study uses factorial analysis, two way ANOVA and polynomial model to analyze the measured data and determine the most significant factors for better performance. The analysis also shows how the factors should be implemented during deployment scenario.

\subsection{Two-way ANOVA}

ANOVA results for the measured values are shown in Figure 6. It can be seen in the figure that $R^{2}$ is $92.35 \%$. This shows that the model is significant. Also, P-value for the height of the node is 0.001 which is less than the assumed alpha level of 0.05 . It means that the height of node is significant in the experiment. Again, the interaction term (power and environment) shows that P-value is low, and this term is also significant.

\subsection{Factorial Design Analysis}

The response values of factorial design experiment are given in Table 2 which is used in factorial design plot like the one in Figure 3. The outcomes are displayed and shown in Figures 7 to 13. The graphs help to analyze the effect of the factors on the performance of wireless sensor network. The software used to plot the graphs does not provide option to represent the figures in vector format. The normal probability plot in Figure 7a shows that these factors are normally distributed. This ensures that there will not be a problem when assuming normality for computation during analysis. Also from Figure 7b, residuals plot are equally distributed about the center line and this shows that the data are structureless which ensures that whatever model formed from these data will be a good and an accurate model. The data satisfies constant variance assumptions. The distribution of RSSI follows Gaussian distribution which is demonstrated in the histogram in Figure 7b. Histogram of residual does not show extreme skewness.

The Pareto charts in Figure 7c are bar charts which allow users to see which factors have statistical significant effects on the performance easily. According to factorial design theory, the bars above the dash-red lines are significant. They describe the magnitude and importance of factor effect. It can be seen from the figure that the height of the node has significant effects on the signal strength. Also, environment and the interaction effects of height and environment are significant. The Normal plot in Figure 8a displays whether the effect of the factor is positive or negative. It can be seen from the figure that height and interaction factor (height and environment) have positive effect on the response as these factors increase in both the theoretical stages and response stages of the graph.

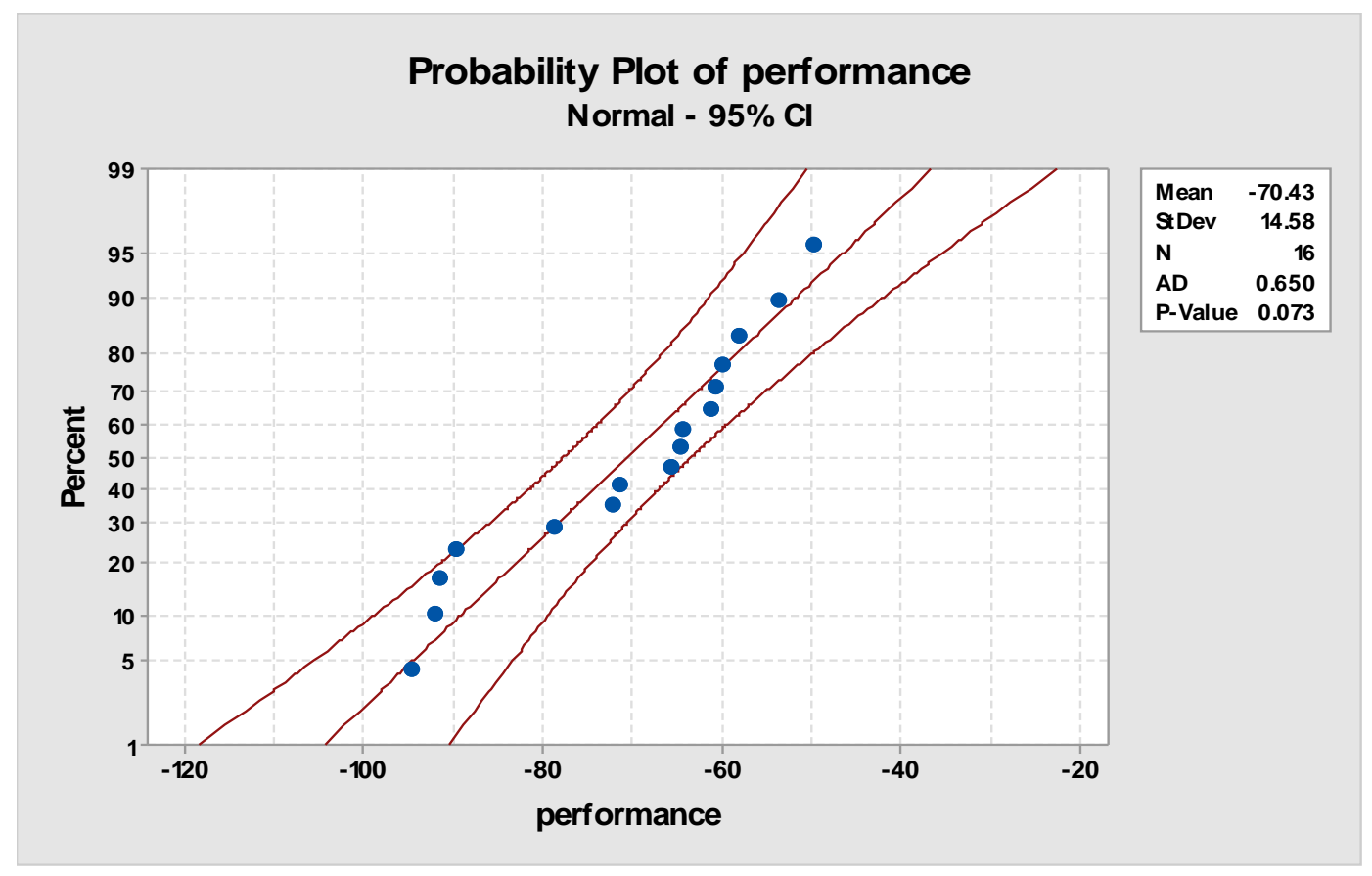

(a) 


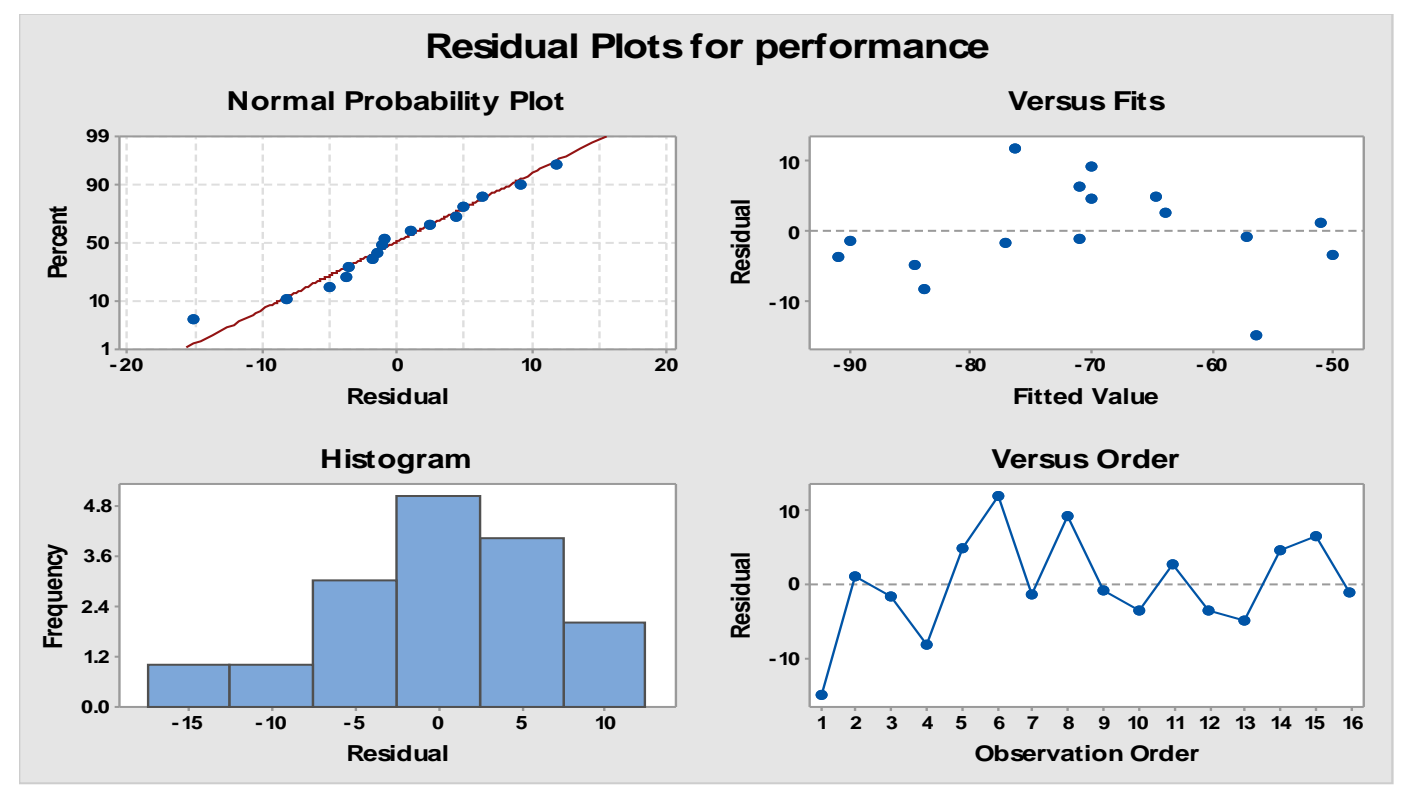

(b)

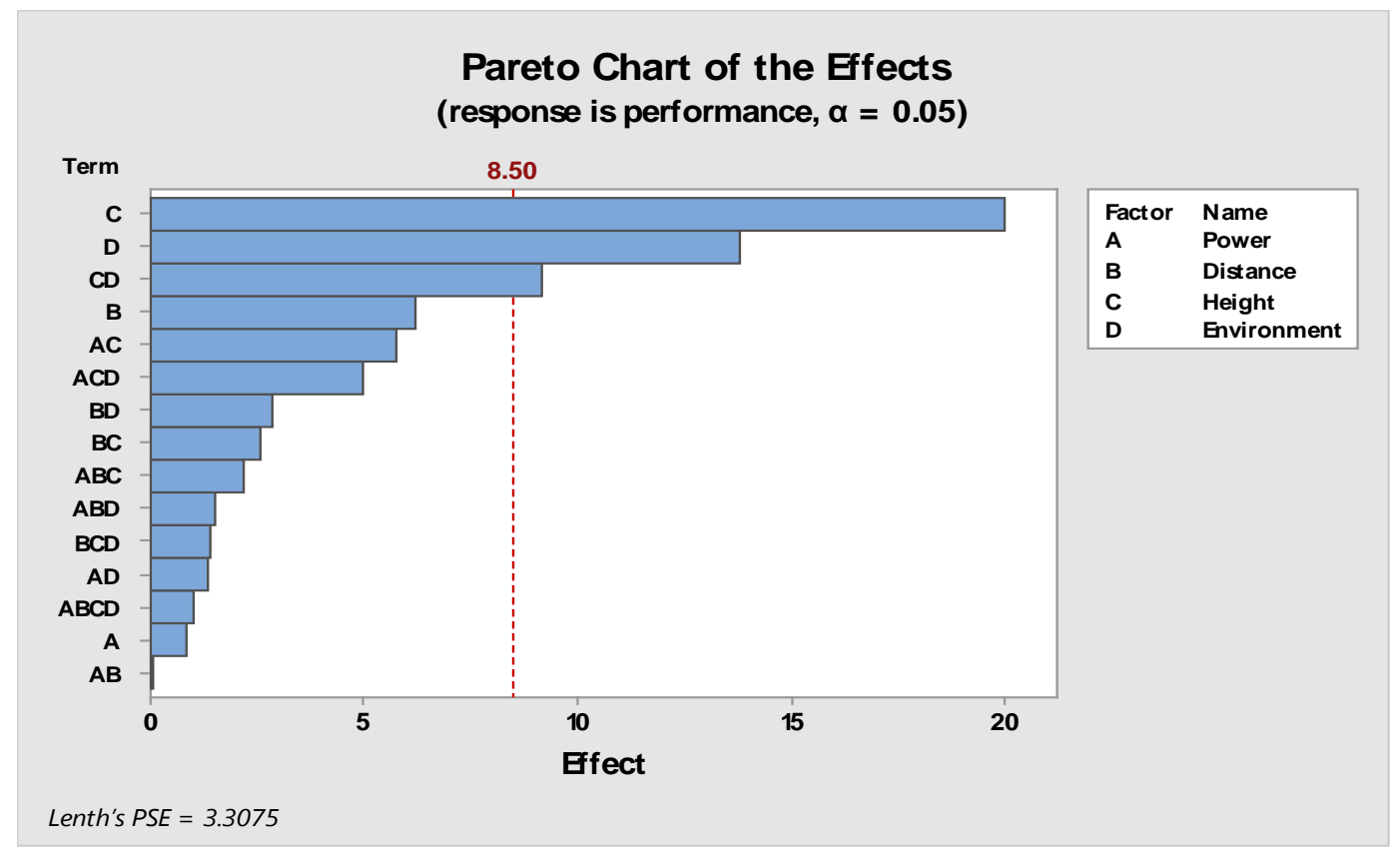

(c)

Figure 7. (a). Normal probability plot, (b). Residual plots, and (c). Pareto Chart of the Standardized factor effects.

Similarly, the Half Normal plot in Figure 8b shows which factors have significant effect on the responses. Height, environment, and interaction factor are shown as red squares; this indicates that these factors have significant effects on the performance of WSN. The factors in blue dots do not have significant effect on the performance. Also, the farther away a factor is from the red line, the more significant effect it has on the corresponding response. From the graph, these two factors: height and environment are further away from the red line. It means they significantly affect the behavior or the performance of wireless sensor network. 


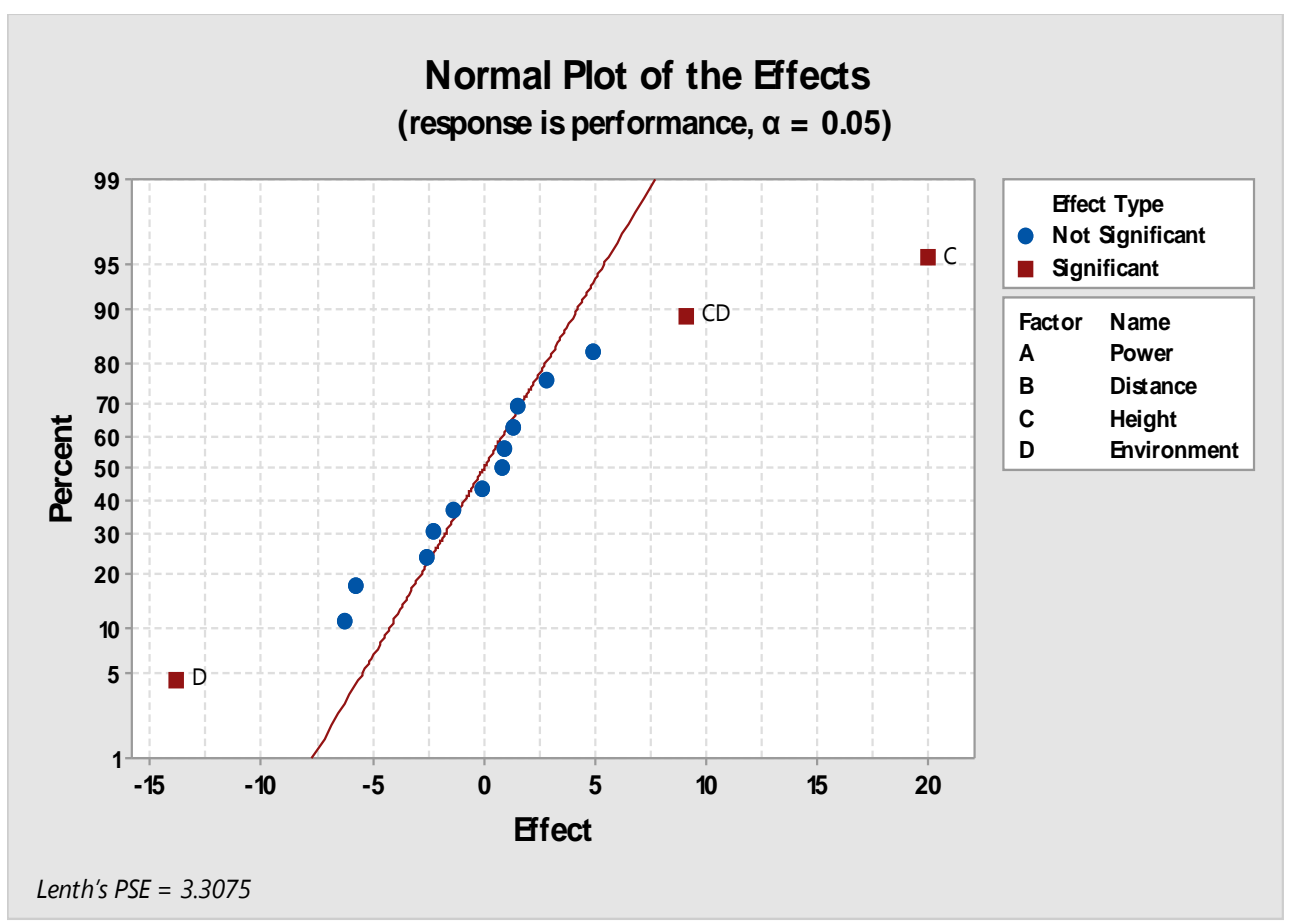

(a)

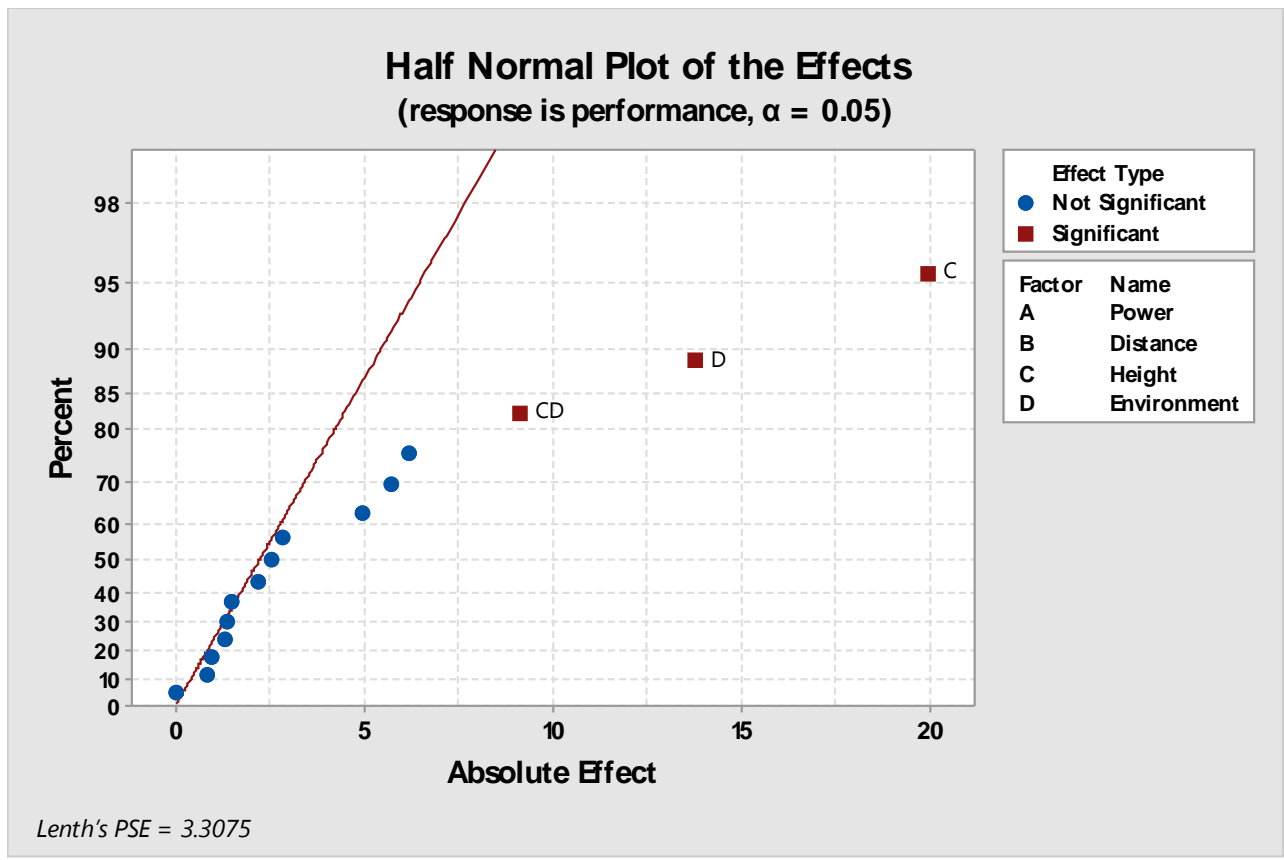

(b)

Figure 8. (a). Normal Plot an, (b). Half Normal Plot of the Standardized Effects of factors

\subsubsection{Factor Main Effect}

From the graph in Figure 9, main effect situation is when there exists a consistent trend among the different levels of factor. This is expressed by the steepness of the lines. Transmission power has little change effect on the signal strength for the experiment. A small increase in distance has negative effect on the wireless sensor network's performance. The height of the node from the ground has the greatest gradient and it will in turn have a positive effect on the node performance. A slight increase in the height of the node above the ground results in strong signal strength for the nodes. The environment also has impact on the node performance. When nodes are in open grass (outdoor), experiment gives positive results than when they are inside the building (indoor).

\subsubsection{Factor Interacting Effects}

In Figure 10, it can be seen that there is interaction 
between power and the environment which is indicated by the crossed lines. For interaction effects of the factors in a design experiment, it is inefficient to describe typical results accurately without mentioning these two factors. Figure 11 shows contour plots for the interactions among the factors. These contours are the projections on factors (for instance in Figure 11a distance-power and Figure 11b environment-power) region of cross sections of the yield surface corresponding to the percentage yields on the response. Each contour corresponds to a particular height of the response surface. There is a large surface to show the effect of these factors. With this factorial design analysis, the region of maximum yield contributes to the better performance.

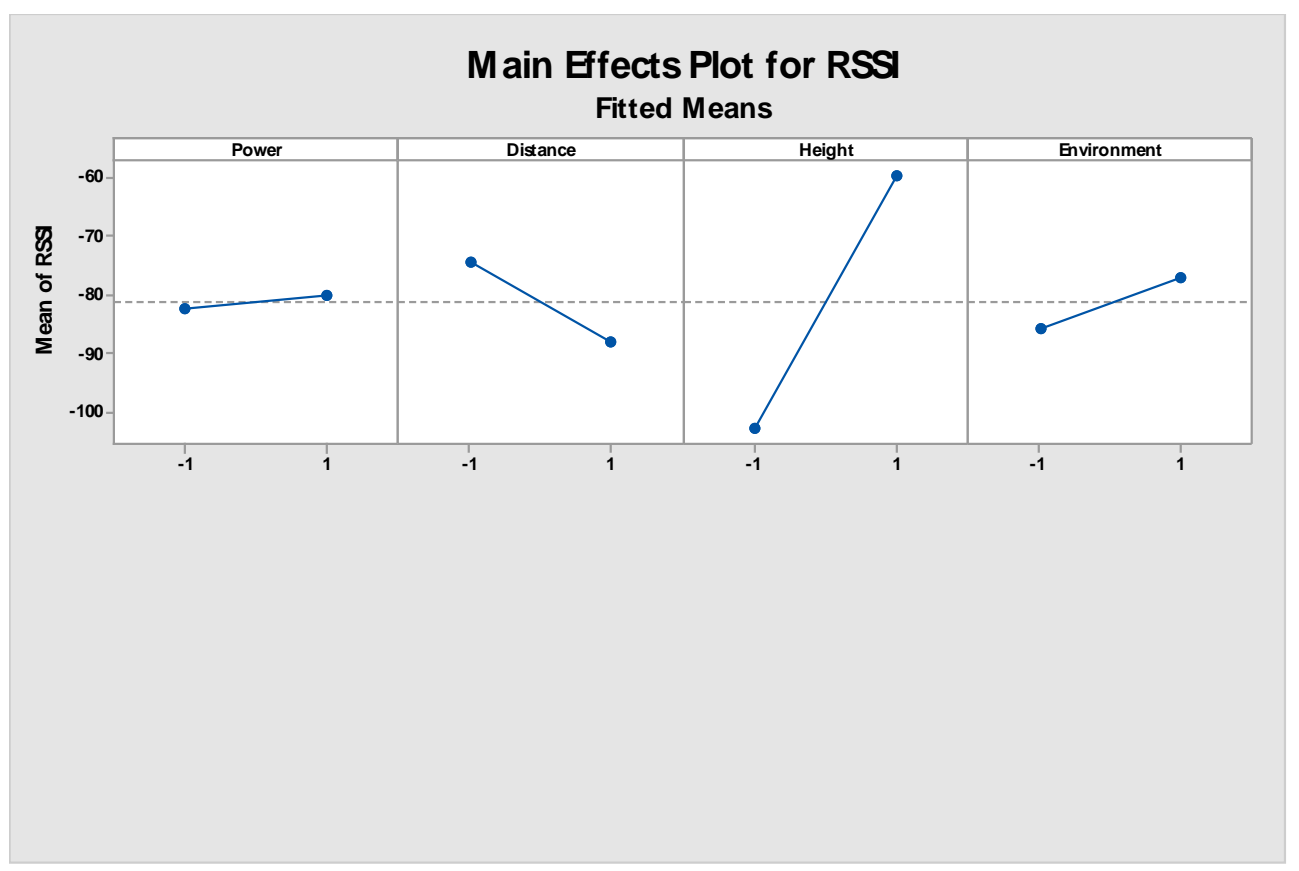

Figure 9. Main effect plots for Received Signal strength indication for Wireless sensor network.

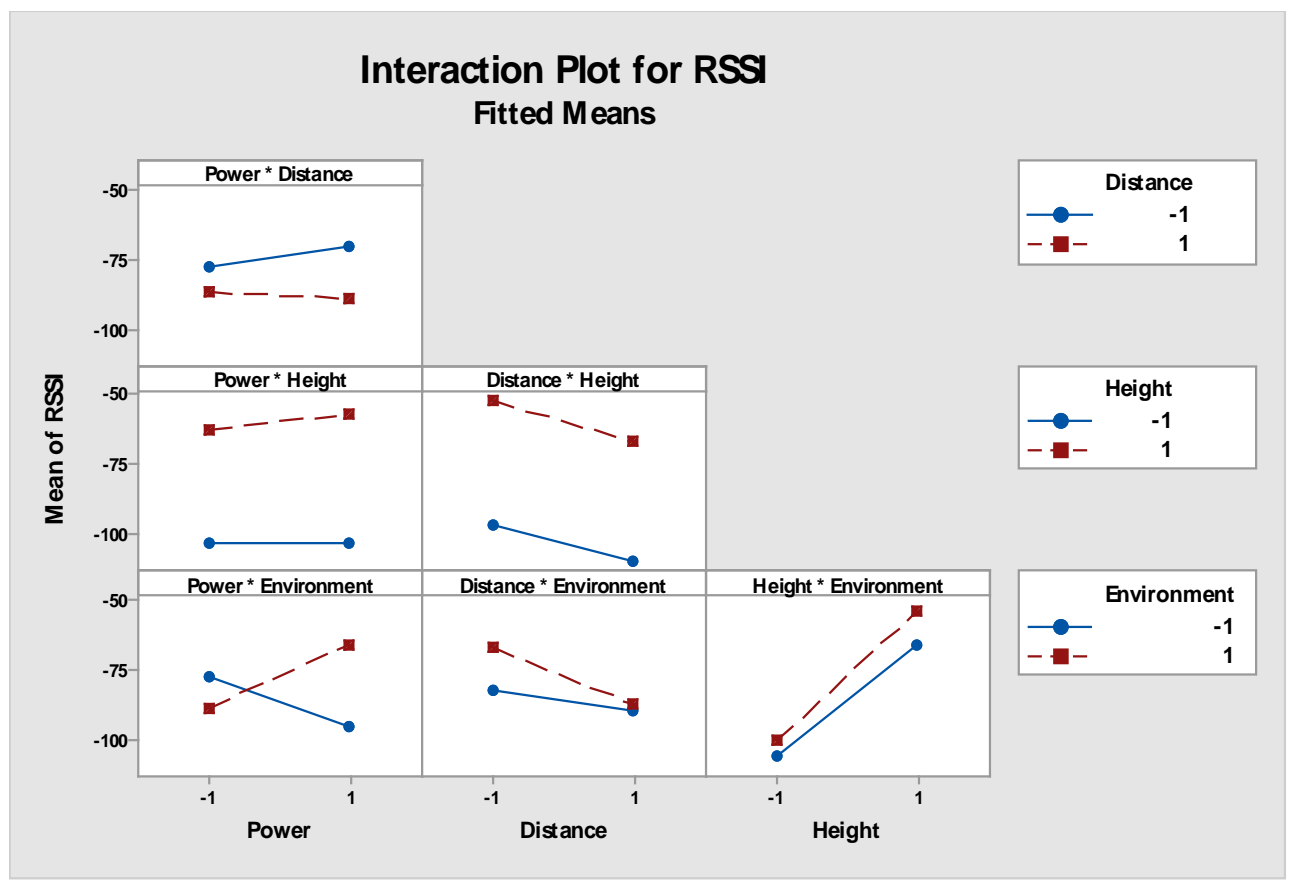

Figure 10. Interaction plots for Received Signal strength indication for Wireless sensor network 


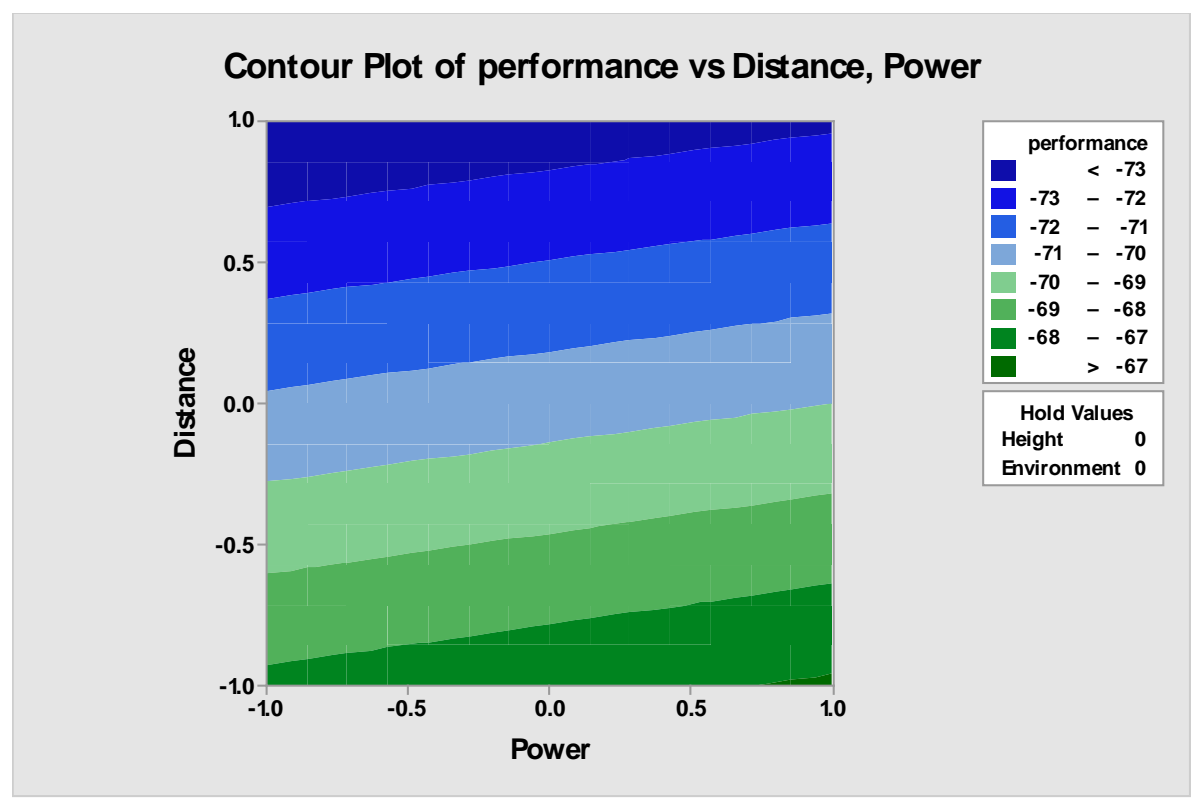

(a)

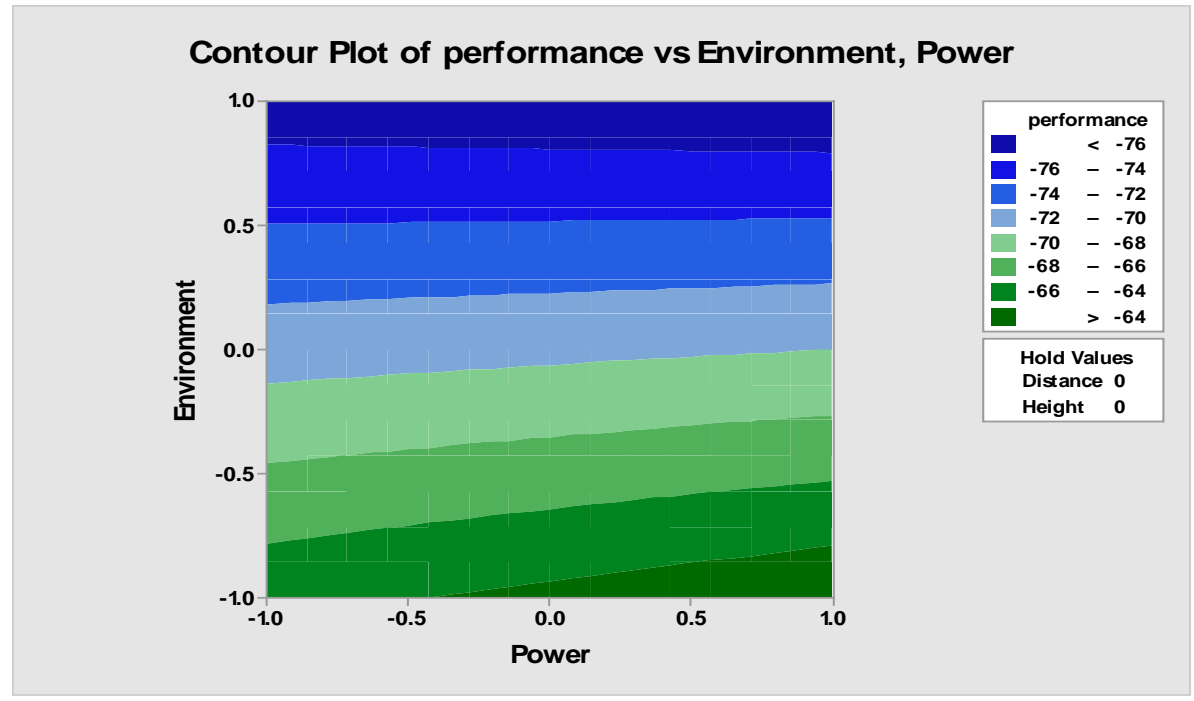

(b)

Figure 11. Response contour plots for interactions among factors: (a). Distance and power, (b). Environment and power 


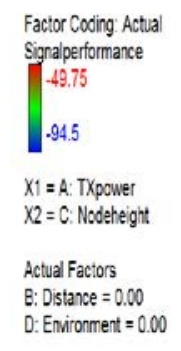

.94 .5

$X 1=$ C: Nodeheight

$X 2=0$. Environment

Actual Factors

A. TXpower $=0.00$

B. Distance $=0.00$

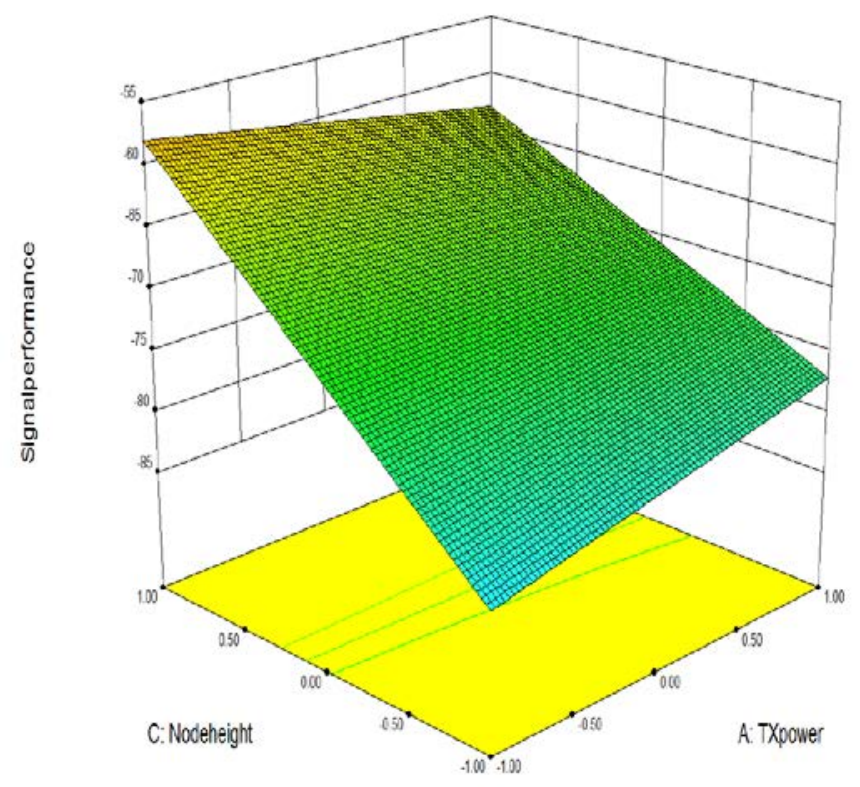

(a)

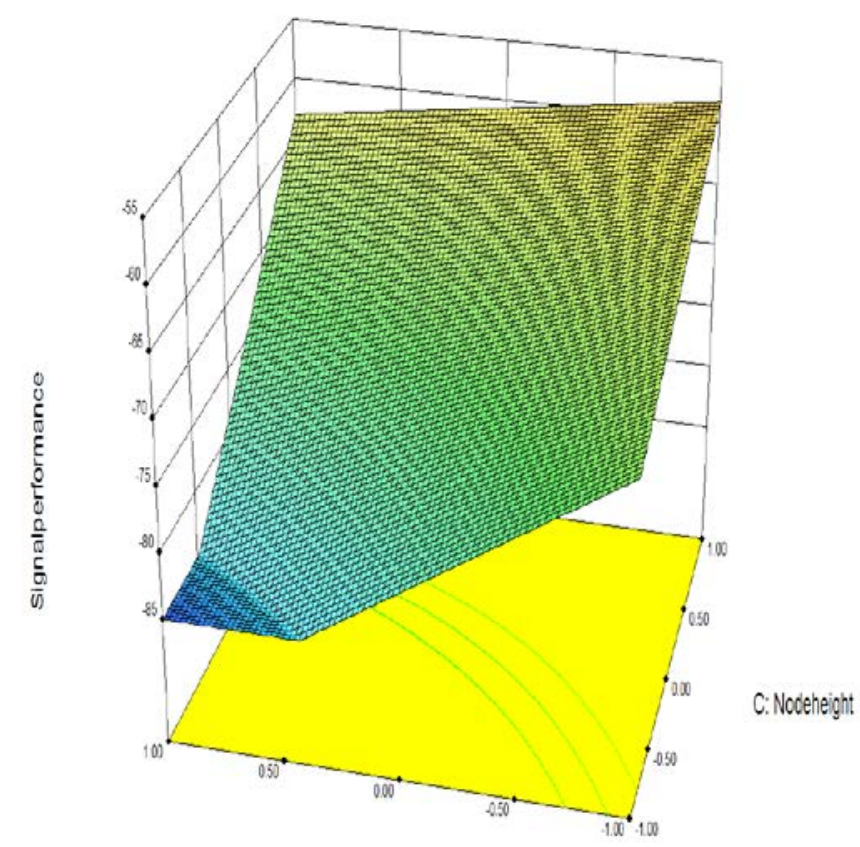

D: Envionment:

(b)

Figure 12. Response surface plots for interactions among factors: (a). Power and height, (b). Environment and height

Figure 12 is a three-dimensional response surface showing the expected response as a function of height and power or environment factor. Since the model contains only the main effect, the fitted response surface is a plane (Figure 3). It has been shown that height, power, and environment together have positive effect on the performance of network. The response surface is used to know which response path is obtained from these factors. As the height of nodes increases, the nodes' performance improves. Method of steepest ascent is used to know the directional potential improvement for this process [15].

\section{3. "Easy Algorithm" for Significant Factors Optimization}

"EASY algorithm" is the steps follow in this study to demonstrate the importance of considering significant factors for an experiment or pre-deployment design for on-demand WSN deployment. It shows a procedure that 
could be used to have knowledge of the performance of the actual network before deployment. Also, it presents the steps to determine the significant factors to be used for such deployment. From the analysis of the factorial experiments, the algorithm uses all factors at initial stage, and beginning to eliminate the least significant factors as the experiment is iterated. The process is repeated until an optimal model is obtained. The algorithm is shown in Figure 13.

\begin{tabular}{|c|c|}
\hline \multicolumn{2}{|c|}{ EASY Algorithm } \\
\hline $1:$ & Input: Combine all factors in experiment not just one \\
\hline $2:$ & for factor level $\rightarrow$ Levels do \\
\hline $3:$ & Initialize operation: do normality test \\
\hline $4:$ & If initial data fit $\rightarrow$ do analysis \\
\hline $5:$ & else $\rightarrow$ do normality test \\
\hline $6:$ & Update $\rightarrow$ select significant factor \\
\hline $7:$ & Update $\rightarrow$ pick best fit model \\
\hline $8:$ & Update $\rightarrow$ validate model \\
\hline $9:$ & end if, end for \\
\hline $10:$ & If model optimal $\rightarrow$ algorithm coverges \\
\hline $11:$ & else $\rightarrow$ go to start \\
\hline $12:$ & end \\
\hline
\end{tabular}

Figure 13. EASY Algorithm

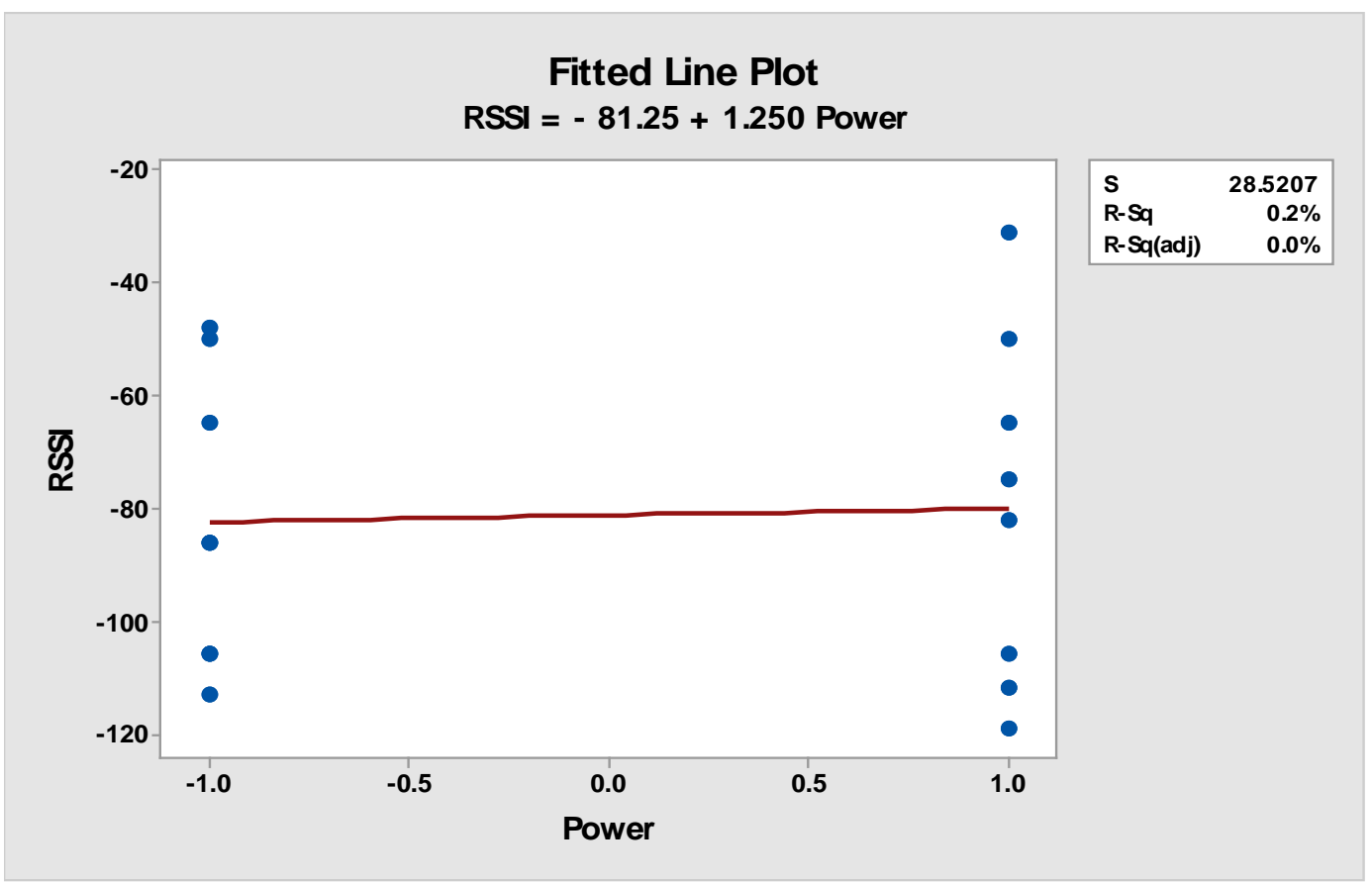

(a) 


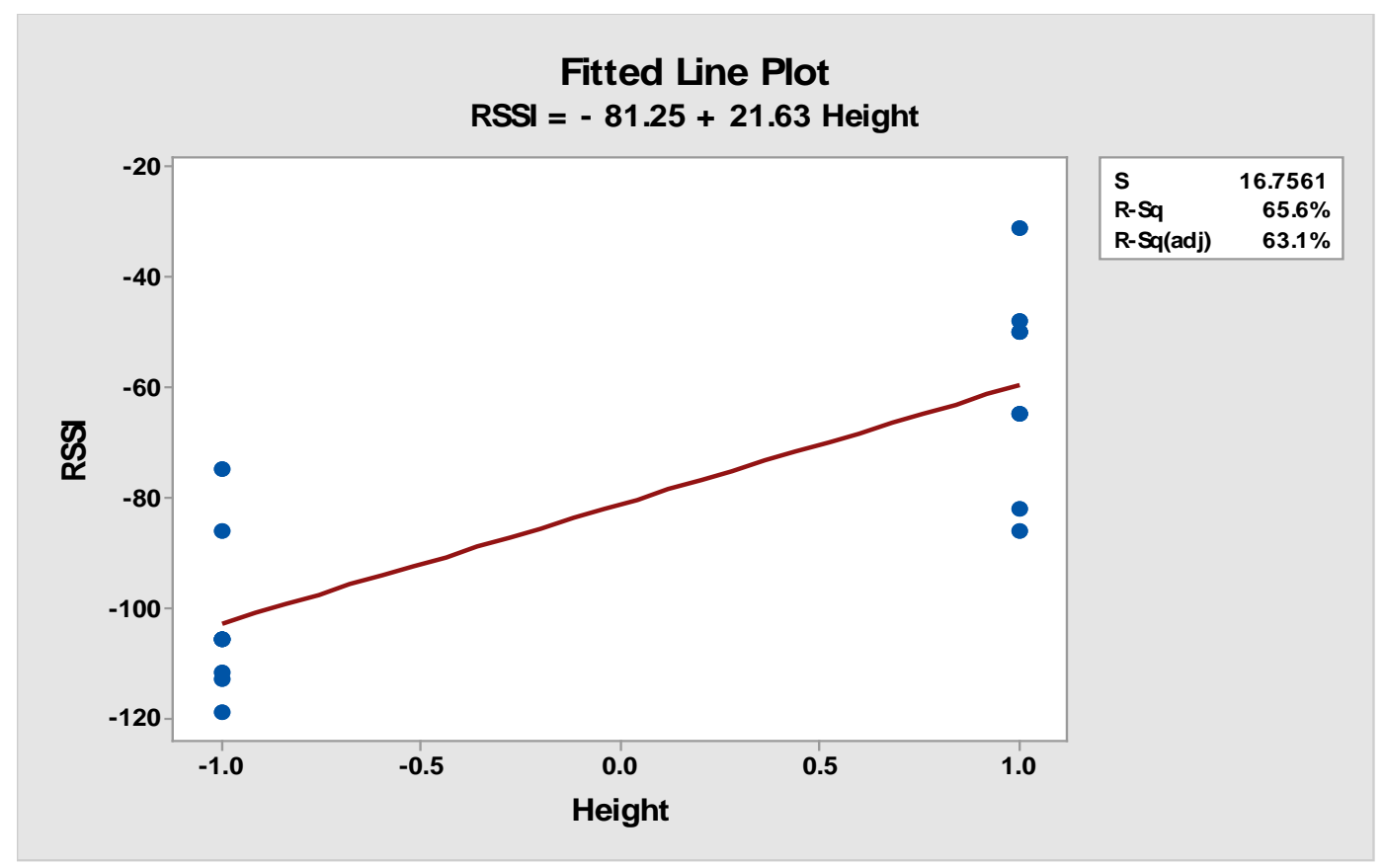

(b)

Figure 14. Regression plots for the analysis of signal performance when one factor is used in the experiment. (a). Signal performance against power (b). Signal performance against height

In the optimization procedure for obtaining the best fit performance model, the best model's significant factors obtained through factorial design analysis discussed earlier are implemented to derive best fit model. This study starts the analysis of regression model by fitting the single factor plot and considering the analysis as shown in Figure 14.

From Figure 14, just one factor- node power or height is used in the analysis, it could be seen from the analysis that p-value for when only height is used in the model is low. Height is significant in the model. The regression model when only height is in the model is

$$
R S S I=-81.25+21.63 \text { Height }
$$

The $R^{2}$ is 0.66 and $R^{2}$ (adjusted) is 0.63 which are good but low. However, F-value is high. The value of $R$ is 0.81 ; this shows that there is a strong relationship between the RSSI measured on the nodes and the heights of the nodes. Using this model will not give optimal performance. The process of finding the optimal polynomial continues until it converges. At convergence, the error approaches zero and hypothesis parameters remain constant. Accordingly, from Figure 6, four factors are considered. The $R^{2}$ is 0.92 and $R^{2}$ (adjusted) is 0.77 , which are better than when considering each one of the other factors in the model. The value of $R$ in Figure 6 is 0.95 . This shows that there is a stronger relationship between the RSSI measured on the nodes and when four factors and interacting terms are included in the model. The p-value for the regression model is 0.03 . This shows that the model could be a good model for future prediction of RSSI values for better performance. The p-value for the height, and interaction term - (power and environment) are 0.001 and 0.028 respectively. It shows that these factors are important in the model. Estimate of standard error improves, which is 13.2. Root mean square error is 5.042. Following the process of the algorithm in Figure 13 and having considered four factors and their interacting effects, the signal level performance optimization model is:

$$
\begin{array}{r}
\text { RSSI }=-81.25+1.25 \text { Power }-6.88 \text { Distance }+ \\
21.63 \text { Height }+4.37 \text { Environment }- \\
2.37 \text { Power } \times \text { Distance }+1.38 \text { Power } \times \\
\text { Height }+10.13 \text { Power } \times \text { Environment }- \\
0.5 \text { Distance } \times \text { Height }-3.25 \text { Distance } \times \\
\text { Environment }+1.50 \text { Height } \times \\
\text { Environment }(11)
\end{array}
$$

Thus, the reduced ANOVA optimization model is given as follows:

$$
\begin{aligned}
& \text { Model }= \\
& -81.25+1.25 \text { Power }+21.63 \text { Height }+ \\
& 10.3 \text { Power } \times \text { Enironment }
\end{aligned}
$$

Factor (power) is included at the first term to maintain hierarchy.

The reduced ANOVA optimization model is able to predict new value with accuracy of $92 \%$ and with error of 5 .

\subsection{Results of Analytical Simulation}

In this study, analytical simulation is performed to show the effect of factors on performance and compare the results with factorial design experiments. The effect of different factors on performance of WSN is simulated. The factors used in the simulation include: node range, environment, coverage area, number of nodes, node distance, and node height. The performance metrics consider for the WSN are connectivity, coverage, and scalability. The nodes are assumed to be randomly deployed at different points and 
distances. Tables 3 to 6 show the factors and connectivity values for simulation.

For all the cases of simulations, sensor nodes are randomly deployed into the selected area. This study simulates the connection between nodes based on Euclidean distance using disc model and count the number of connectivity. Node random placement uses Poisson model Agrawal et al. [34]. The approach is to generate random points $A\left(x_{1}, y_{1}\right)$, $B\left(x_{1}, y_{1}\right)$, and so on to represent the location of nodes. A connection is set up between 2 or among more nodes using the Euclidian distance $\left(E_{d}\right)$ between the points. If the $E_{d}$ is less than or equal to the node range, there is connection between two or among more nodes, otherwise there is no connection. From Cartesian coordinates and using Pythagoras formula, $E_{d}$ between points $D\left(x_{1}, x_{2}, \ldots x_{n}\right)$ and $E\left(y_{1}, y_{2}, \ldots y_{n}\right)$ is

$$
=\sqrt{\sum_{j=1}^{n}\left(x_{j}-y_{j}\right)^{2}}
$$

The connectivity model states that a sensor node is connected to its neigbor if the $E_{d}$ between them is less than or equal to twice the sensing range of node.

Mathenatially, connecticity model is given as:

$$
r_{c}=E_{d} \leq 2 r_{s}
$$

where $r_{c}$ is the connectivity range and $r_{s}$ is the sensor or radio range.

The simulation shows the effects of various factors on the connectivity and scalability performance of random wireless sensor networks deployment. In Figures. 15 to 28, the green solid circle denotes the node, the red line is the connectivity or communication range, and the blue big circle represents the node sensing range. The Cartesian graph symbolizes the deployment area. The effect of irregular environment or terrain (3D) is modeled into the simulation with different environments Olasupo et al. [35]. For better visualization of problem at hand, this study does not show the 3D space or height

Table 3. Number of Connectivity in Different Deployment Dimensions

\begin{tabular}{|c|c|c|}
\hline Deployment Area (m x m) & $\begin{array}{c}\text { No of node } \\
\text { connectivity }\end{array}$ & Coverage area \% \\
\hline $200 \times 200$ & 417 & 100 \\
\hline $400 \times 400$ & 154 & 38.50 \\
\hline $600 \times 600$ & 84 & 14.00 \\
\hline $800 \times 800$ & 53 & 6.60 \\
\hline $1000 \times 1000$ & 38 & 3.80 \\
\hline $1200 \times 1200$ & 28 & 2.30 \\
\hline $1400 \times 1400$ & 21 & 1.50 \\
\hline $1600 \times 1600$ & 13 & 0.88 \\
\hline $1800 \times 1800$ & 9 & 0.50 \\
\hline $2000 \times 2000$ & 5 & 0.125 \\
\hline
\end{tabular}

Table 4. Number of Connectivity in Different Environments and Environment Type is Simulated using the Models in [10] and Olasupo et al. [36]

\begin{tabular}{|c|c|}
\hline Environment type & Number of node connectivity \\
\hline Sparse tree & 51 \\
\hline Long natural grass & 530 \\
\hline Artificial turf grass & 94 \\
\hline Sandy terrain & 40 \\
\hline Short natural grass & 174 \\
\hline Concrete surface & 41 \\
\hline Dense tree & 28 \\
\hline
\end{tabular}

Table 5. Number of Connectivity at Various Node Ranges

\begin{tabular}{|c|c|}
\hline Node Range & No of node connectivity \\
\hline 100 & 2 \\
\hline 200 & 6 \\
\hline 300 & 15 \\
\hline 400 & 16 \\
\hline 500 & 23 \\
\hline 600 & 30 \\
\hline 700 & 35 \\
\hline 800 & 38 \\
\hline 900 & 40 \\
\hline 1000 & 44 \\
\hline
\end{tabular}

\section{Case 1}

This case describes connectivity performance at different deployment dimensional areas. 50 nodes at $100 \mathrm{~m}$ range are randomly deployed in the same environment for all scenarios. Table III shows the number of node connectivity. Figures 15 to 17 show the connectivity plots and Figure 18 shows the performance of the deployments.

Table 6. Number of Connectivity at Different Number of Nodes

\begin{tabular}{|c|c|}
\hline Number of node & Number of node connectivity \\
\hline 10 & 2 \\
\hline 20 & 3 \\
\hline 30 & 11 \\
\hline 40 & 24 \\
\hline 50 & 38 \\
\hline 100 & 122 \\
\hline 200 & 404 \\
\hline 300 & 820 \\
\hline 400 & 1395 \\
\hline 500 & 2151 \\
\hline 600 & 3171 \\
\hline 700 & 4138 \\
\hline
\end{tabular}




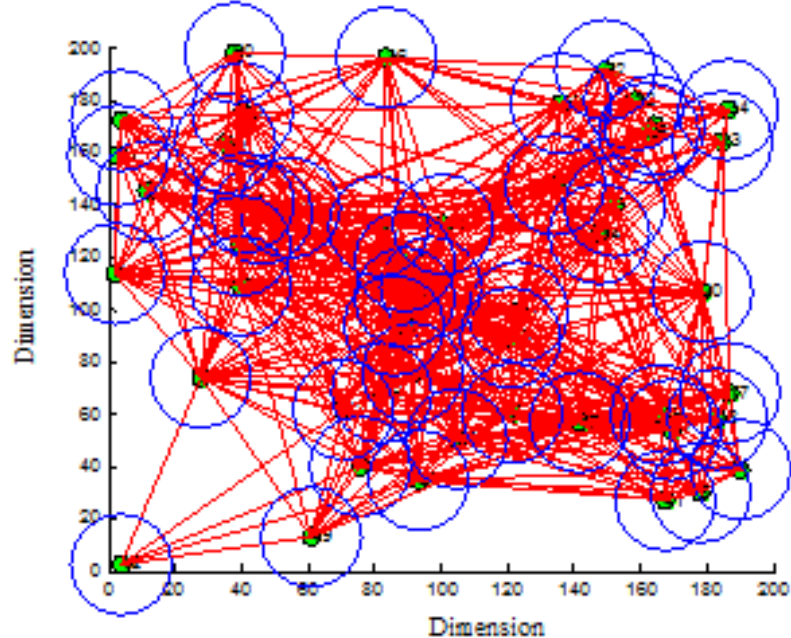

Figure 15. Performance for Area $=200 \mathrm{~m}$ x $200 \mathrm{~m}$, range $=100 \mathrm{~m}$, number of node $=50$, same environment, at random distance for random deployment.

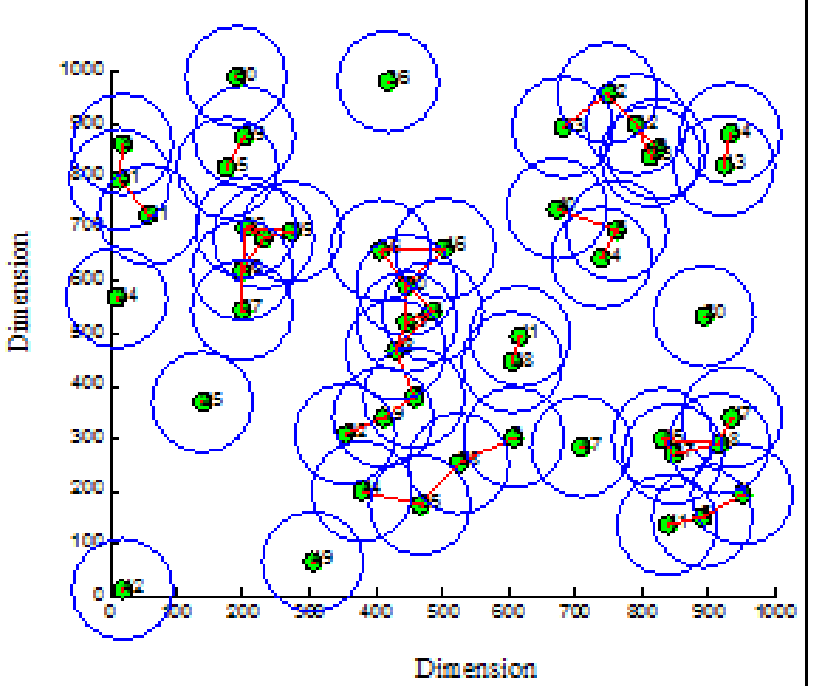

Figure 16. Performance for Area $=1000 \mathrm{~m} \times 1000 \mathrm{~m}$, range $=100 \mathrm{~m}$, number of node $=50$, same environment, at random distance for random deployment.

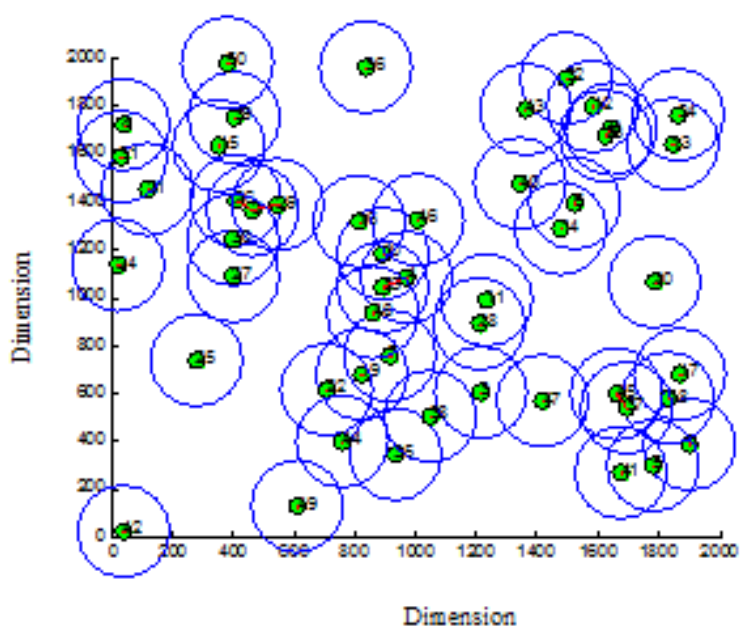

Figure 17. Performance for Area $=2000 \mathrm{~m} \times 2000 \mathrm{~m}$, range $=100 \mathrm{~m}$, number of node $=50$, same environment, at random distance for random deployment

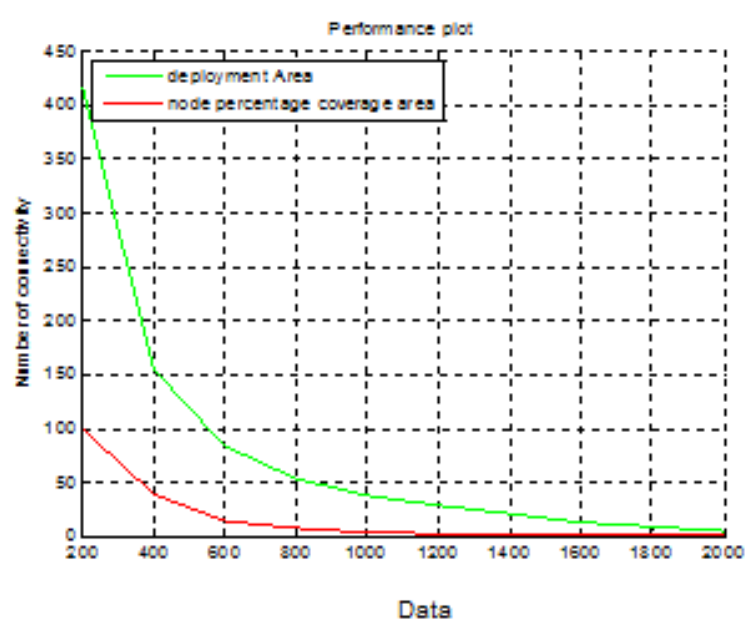

(a)

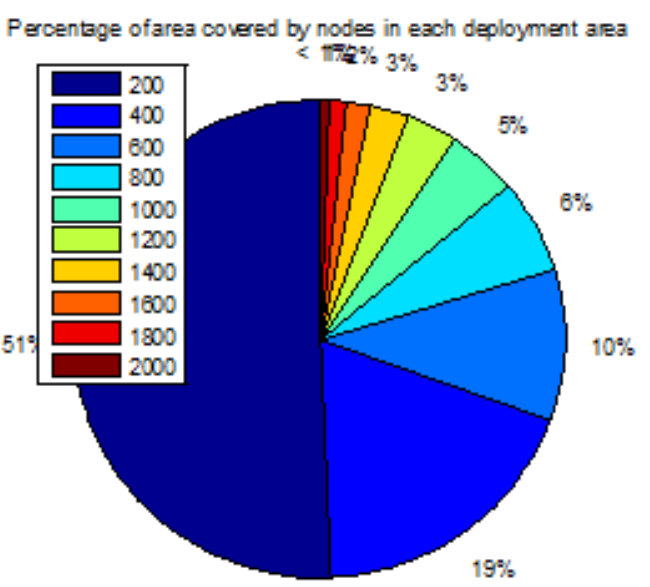

(b)

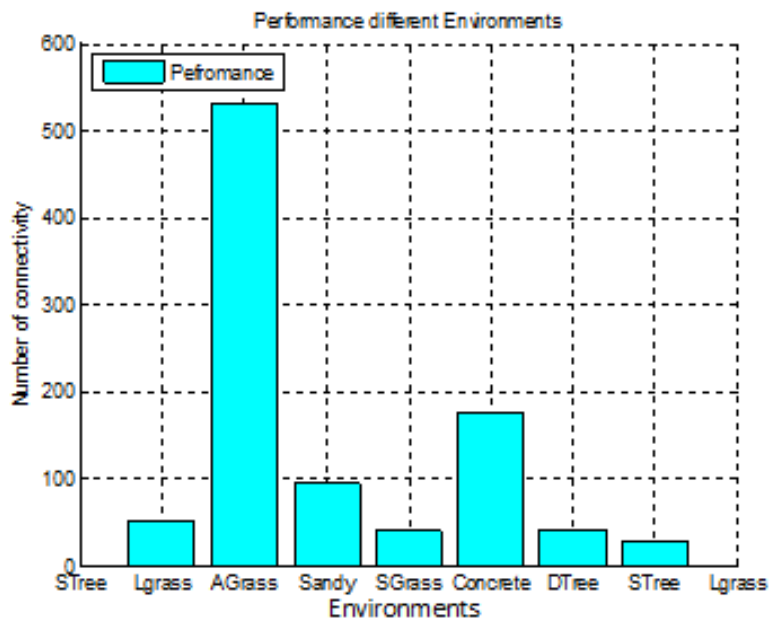

(c)

Figure 18. (a) - (b) Percentage coverage per deployment area and (c) Variation of connectivity due to different environments.

Using Table 3, Figures 15 to 17, this study analyzes the performance metric. It is shown that as the deployment area is becoming larger, more nodes are required to cover the 
entire area. With more nodes, there is a chance for better connectivity performance. Also, it gives opportunity to have good node sensing and communicating range. However, there will be increase in the cost of node procurement. Figures. 14 a-b show the percentage of node coverage per deployment area. Coverage metrics degrade as the deployment area increases.

\section{Case 2}

This case describes connectivity performance at different environments. 50 nodes at $100 \mathrm{~m}$ range are randomly deployed on the same area size for all scenarios. Table 4 shows the number of node connectivity. Environment type is simulated using the models in [12].

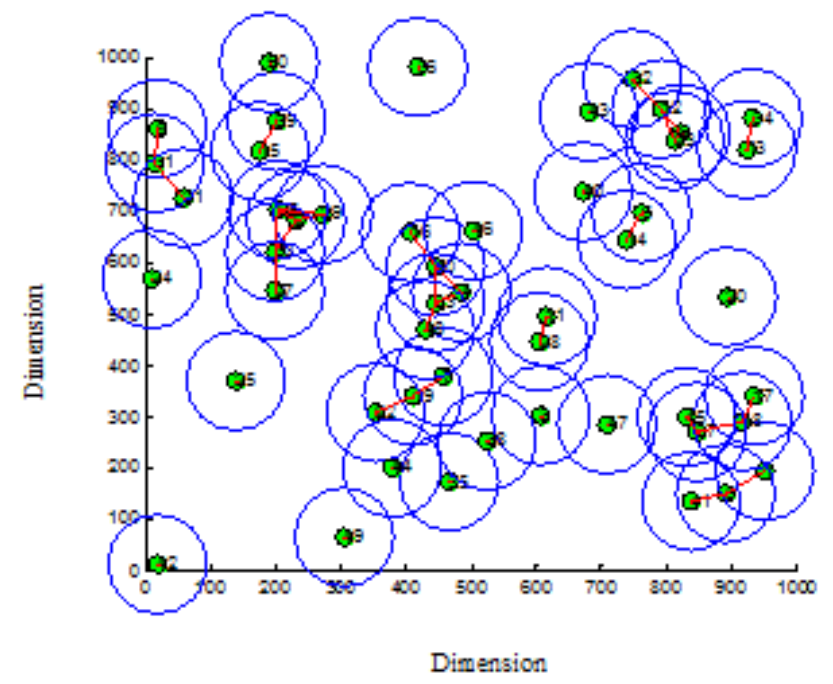

Figure 19. Performance for Area $=1000 \mathrm{~m} \times 1000 \mathrm{~m}$, range $=100 \mathrm{~m}$, number of node $=50$, Dense tree environment

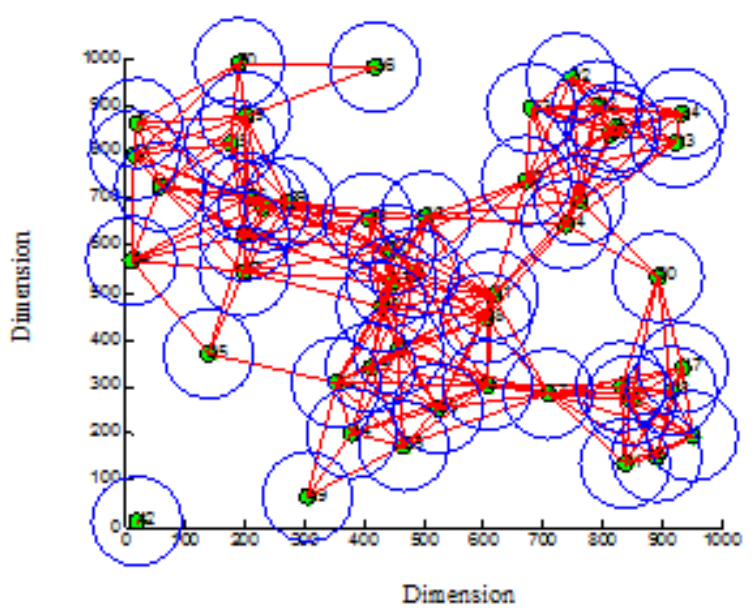

Figure 20. Performance for area $=1000 \mathrm{~m} \times 1000 \mathrm{~m}$, range $=100 \mathrm{~m}$, number of node $=50$, short natural grass environment

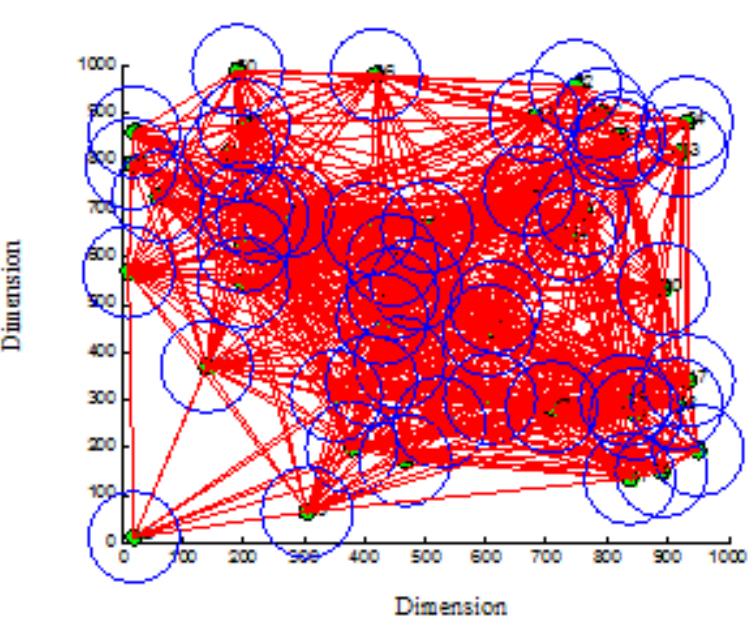

Figure 21. Performance for area $=1000 \mathrm{~m} \times 1000 \mathrm{~m}$, range $=100 \mathrm{~m}$, number of node $=50$, long natural grass environment

Figures 19 to 21 show the analysis of case 2 scenario. Connectivity and coverage performance in dense tree and rough mountainous environment is poor. This is as a result of terrain variants and topology in this environment Olasupo et al. [35], Olasupo et al. [37]. The performance metric is better in low and tall flat terrain grass.

\section{Case 3}

This case describes connectivity performance at different node ranges. Number of node deployed at random distances is 10 . Deployment area of $1000 \mathrm{~m} \times 1000 \mathrm{~m}$ is used in the same environment. Table 5 shows the number of node connectivity.

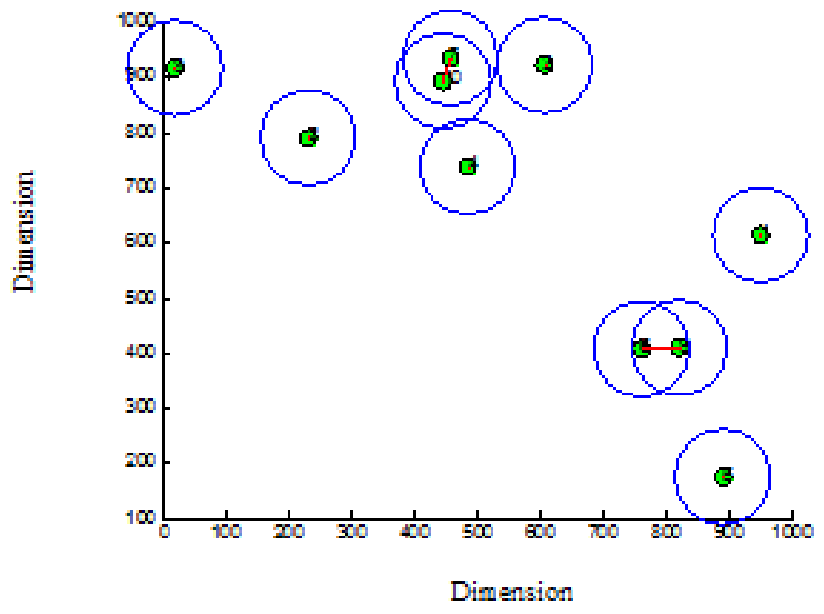

Figure 22. Performance for area $=1000 \mathrm{~m} \times 1000 \mathrm{~m}$, range $=100 \mathrm{~m}$, number of node $=10$, same environment 


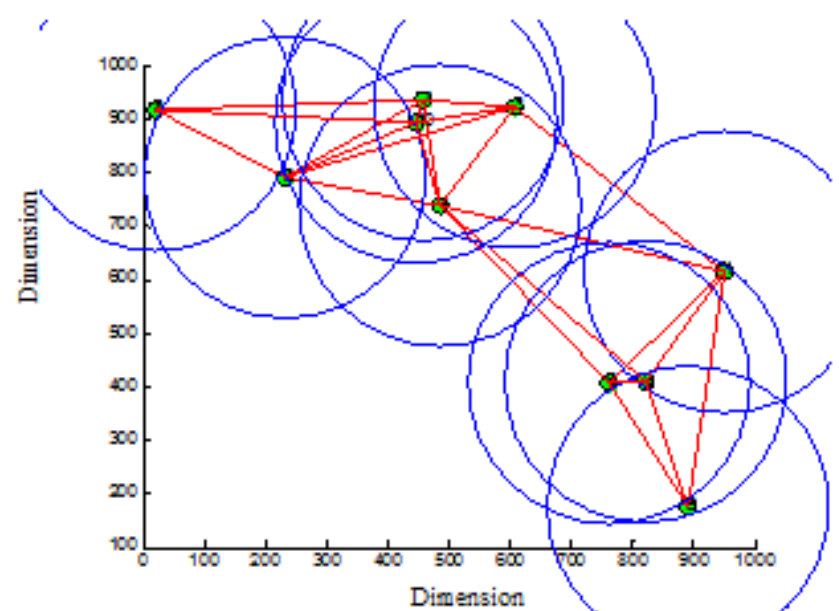

Figure 23. Performance for area $=1000 \mathrm{~m} \times 1000 \mathrm{~m}$, range $=500 \mathrm{~m}$, number of node $=10$, same environment

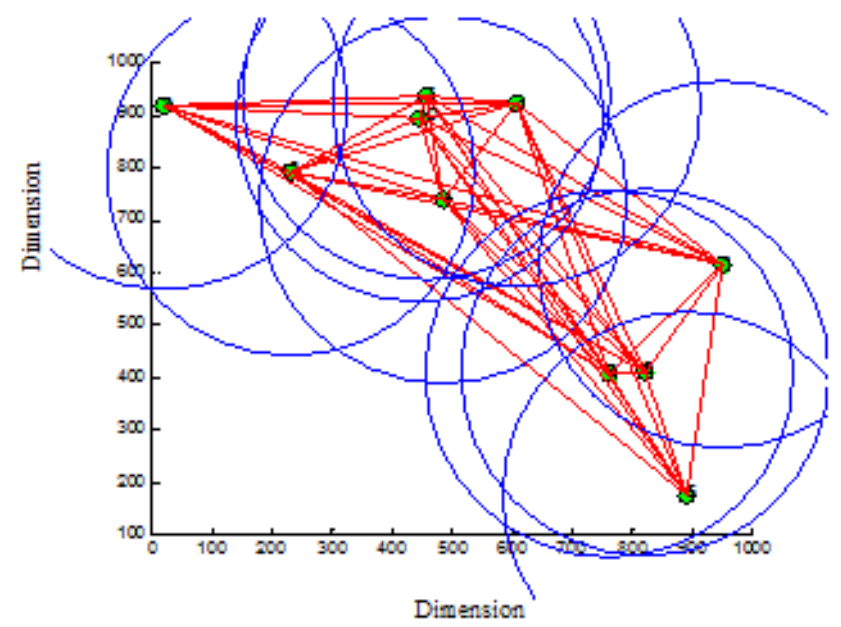

Figure 24. Performance for area $=1000 \mathrm{~m} \times 1000 \mathrm{~m}$, range $=1000 \mathrm{~m}$, number of node $=10$, same environment

Figures 22 to 25 show the analysis plots of case 3. Increase in the communication and sensing range increases the connectivity and coverage performance. Therefore, these will improve the reliability and availability performance metric of the network. Conversely, to increase range means to increase the network cost of transmission power and node hardware. It may also cause interference to neighboring network [4].

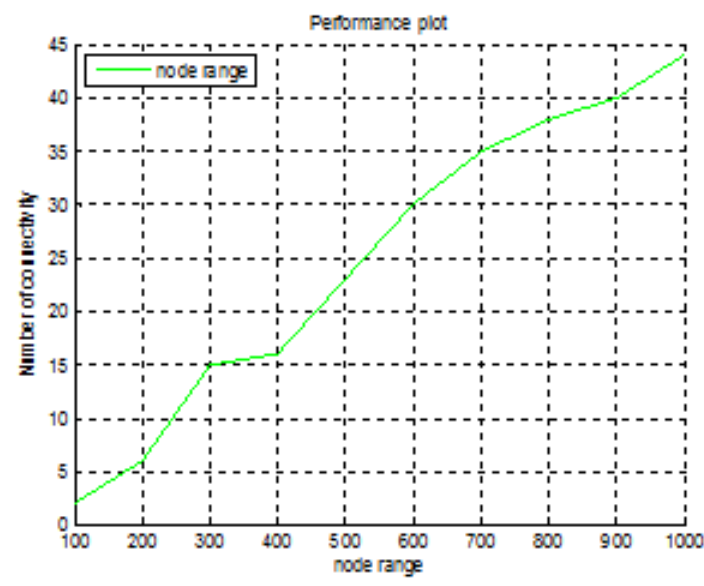

(a)

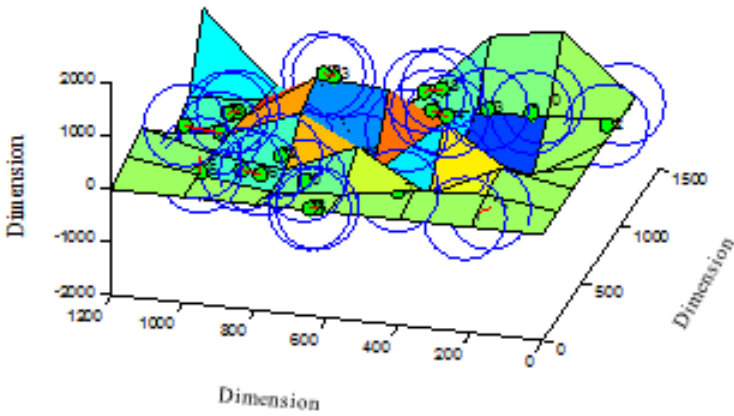

(b)

Figure 25. (a) Performance plot for connectivity and node range, (b) Performance due to terrain height and obstruction

\section{Case 4}

This case describes connectivity performance for different numbers of nodes at $100 \mathrm{~m}$ range. Deployment area of $1000 \mathrm{~m}$ $\mathrm{x} 1000 \mathrm{~m}$ is used in the same environment. Table 6 shows the number of node connectivity. Figures 22 to 29 show the performance metric in case 4 . When there is increase in the number of nodes, the network gives good connectivity and coverage performance metric. This in turn reduces the cost of transmission. However, it can be shown that there are empty spaces or holes, redundant and overlapping nodes due to wrong positioning in the stochastic deployment. Also, the cost of node procurement increases immensely. 


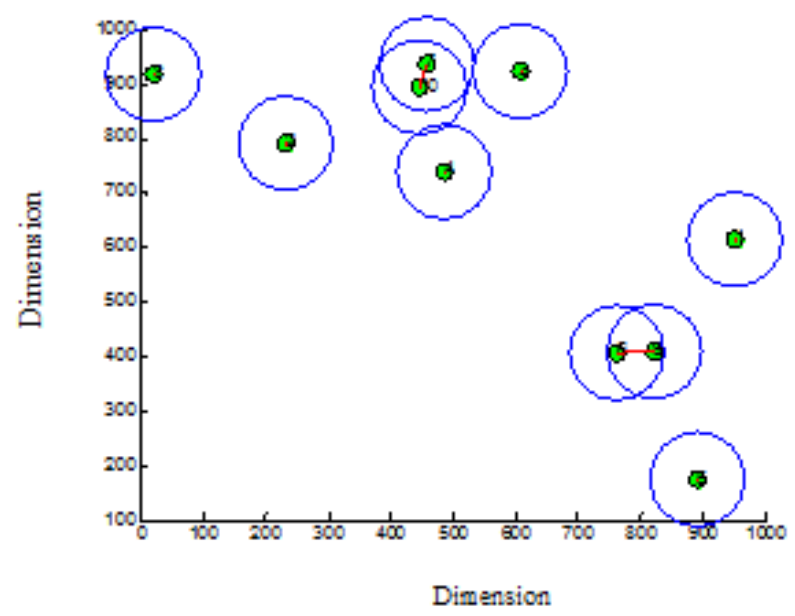

Figure. 26. Performance for area $=1000 \mathrm{~m} \times 1000 \mathrm{~m}$, range $=100 \mathrm{~m}$, number of nodes $=10$, same environment.

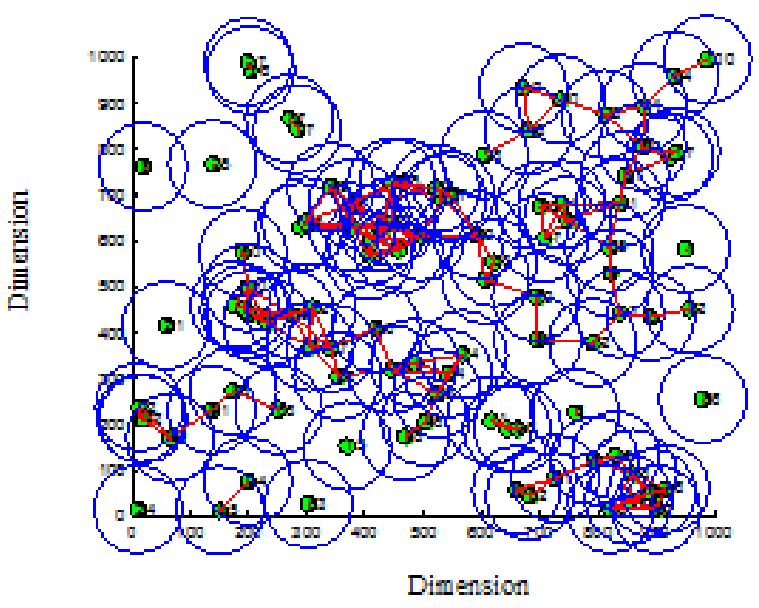

Figure 27. Performance for area $=1000 \mathrm{~m} \times 1000 \mathrm{~m}$, range $=100 \mathrm{~m}$, number of nodes $=100$, same environment.

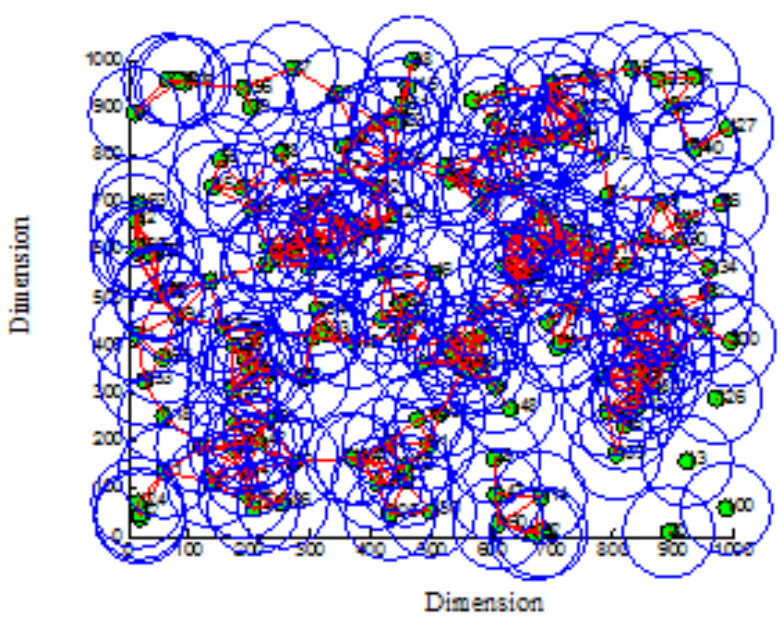

Figure 28. Performance for area $=1000 \mathrm{~m} \times 1000 \mathrm{~m}$, range $=100 \mathrm{~m}$, number of nodes $=300$, same environment.

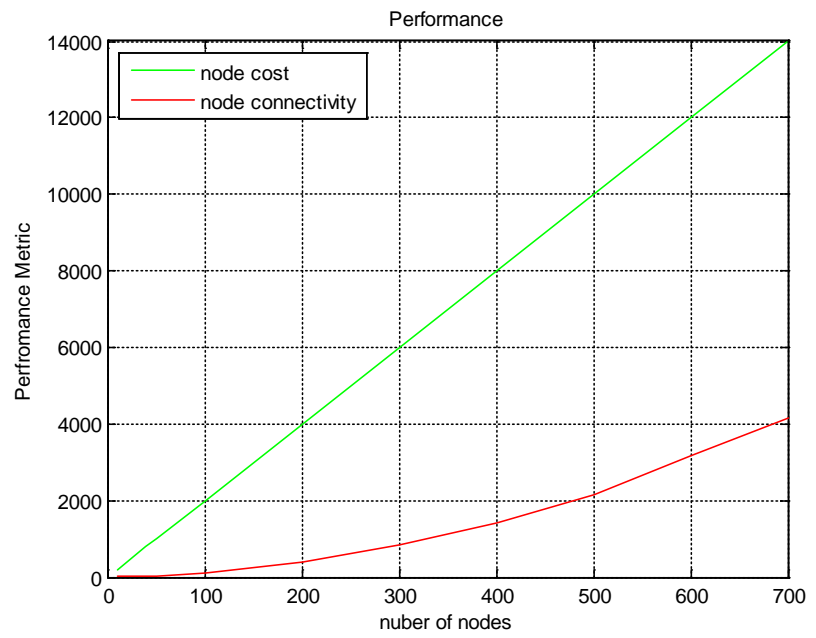

Figure 29. Cost of node versus connectivity (cost of node is $\$ 20$ per piece).

\subsection{Performance Evaluation of Optimization Model}

Having considered both simulation and experimental analysis of factors that determine WSN performance, this study compares both results.

Figure 30 shows the variation of performance metrics when significant factors and their interacting effects are considered during simulation design. The ground truth is the reference or already known solution set for the simulation algorithm. When considering more than two significant factors, the accuracy is $92 \%$ as compared to the ground truth. This study is further validated by comparing the result of the simulation approach with experimental approach. Experimental approach and simulation approach show similar results for deployment decision proposal as shown in Figure 31. However, the study assumes large-scale deployment in the simulation approach. The simulation results are further compared with, and they are similar to the studies in [29-41].

\subsubsection{Factor Main Effect}

From the graph in Figure 9, main effect situation appears when there exists a consistent trend among the different levels of factor. This is expressed by the steepness of the lines.

Based on the results obtained in this study, using only one significant factor in a design yields $R^{2}$ of 0.66 . Whereas, the result gives $R^{2}$ of 0.92 when more than two significant factors are considered.

For optimization deployment of WSN, maximum attainable height of the node should be used in order to minimize the residual or poor performance. In addition, other significant factors and their interaction effects based on performance metrics should be used. The transmission power and the environment should be carefully selected to have a good network performance. The transmit power should not be too low, otherwise there will be poor coverage or too high, otherwise it will drain the power of the node or 
overshadow nearby network. The effect of choice of transmit power can be modeled into the link budget. Finally, the nodes should not be too far away from one another to minimize the negative effect of increase in distance. However, these recommendations for WSN deployment optimization are applications dependent and the global and local minimum depend on the factor types and their factorial design analysis.

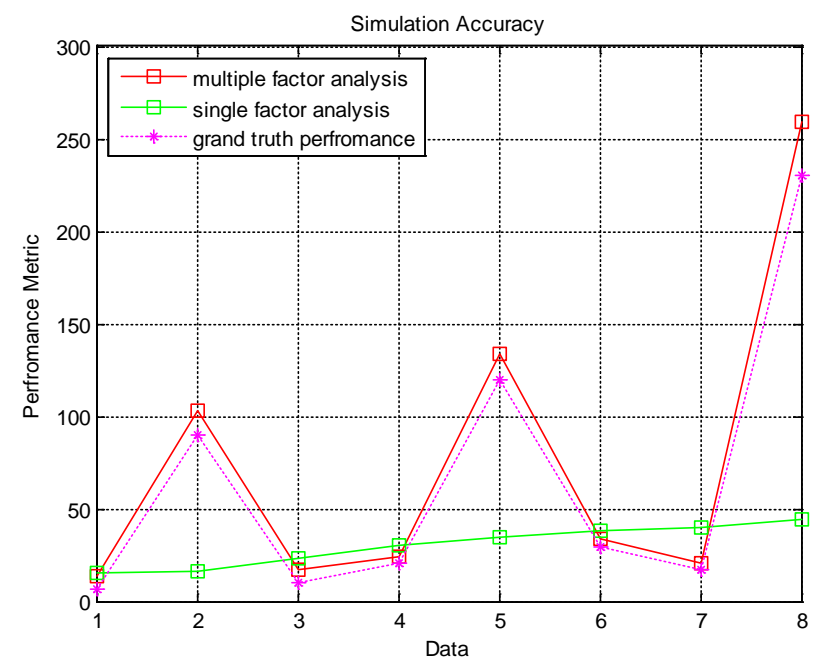

Figure 30. Performance and simulation validation

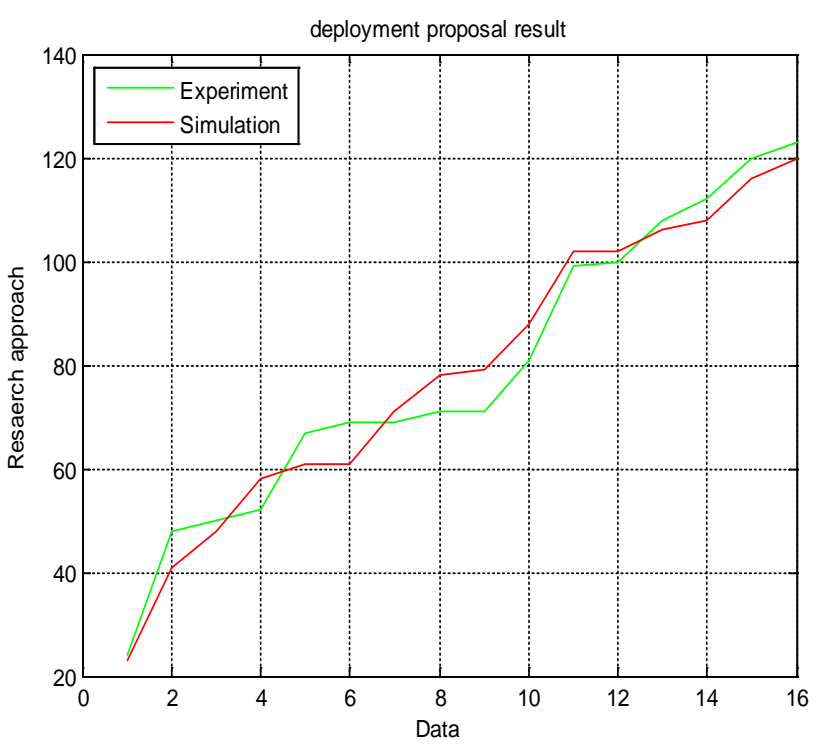

Figure 31. Deployment decision proposal validation.

\section{Conclusions}

This study shows by experiment and simulation that node height, node distance, node power transmission, deployment dimensional area, and environment where nodes are deployed affect the performance of node deployment. It has been shown from the results of the experimental and simulation analysis that when choosing operating conditions for wireless sensor network design, these factors, and their interaction effects have to be taken into consideration. The results show that the inclusion of more significant factors in performance model will optimize the model by $26 \%$ (from $66 \%$ to $92 \%$ ).

A thorough simulation and visualization analysis of the contributions of various assumed factors can help to make a trade off or vital decision among many performance metrics especially for large-scale stochastic deployment where practical experiment analysis is not feasible. This study has shown that the performance metric result is positive when one most significant factor is used in a design or system process, but such design does not give optimum result as futuristic predictor.

Further research will be the study of wireless sensor network performance at different deployment environments using different methodologies. Also, simulation and deployment models that will characterize these environments for scalable large-scale deployment could be studied. Moreover, experimental measurements and empirical models that characterize these environments could be conducted.

\section{REFERENCES}

[1] G. J. Fernandez, I. Cuinas, M. G. Sanchez and V. A. Alejos, "Radio-Electric Validation of an Electronic Cowbell Based on ZigBee Tchnology," Antennas and Propagation Magazine, IEEE, vol. 53, no. 4, pp. 40-44, 2011.

[2] J. A. Gay-Fernandez and I. Cuinas, "Peer to Peer Wireless Propoagtion Measurements and Pathloss Modeling in Vegetation Environments," IEEE Transactions on Antenna and Propagation, vol. 61, no. 6, pp. 3302-3311, 2013.

[3] P. K. Chong and D. Kim, "Surface_Level Path Loss Modeling for Sensor Network in Flat and Irregular Terrain," ACM Transaction on Sensor Networks, vol. 9, no. 2, 2013.

[4] H. Karl and A. Willig, Protocol and Architectures for Wireless Sensor Networks, John Willey \& Son, Inc, 2012.

[5] I. F. Akyildiz, Y. Sankarasubramaniam and E. Cayirci, "Wireless sensor networks: a survey," Elsevier Science B.V, pp. 393-422, 2002.

[6] I. F. Akyildiz, Wireless Sensor Network, Wliey, 2010.

[7] D. L. Ndzi, M. A. Arif, A. M. Shakkaf, M. N. Ahmad, M. F. Ramli and M. S. Razalli, "Signal Propagation Analysis for Low Data Rate Wireless Sensor Network Applications in Sport Grounds and on Roads," Progress in Electromagnrtics Research Journal, vol. 125, no. 1, pp. 1-19, 2012.

[8] A. Harun, M. F. Ramli, D. L. Ndzi, L. M. Kamarudin, M. N. Jaafar and A. Zakaria, "Antenna Positioning Impact on Wireless Sensor Networks Deployemnts in Agriculture," Austarlian Journal of Basic and Applied Science, vol. 7, no. 5, pp. 55-60, 2013.

[9] H. Liu, Z. Meng and Y. Shang, "Sensor Nodes Placement for Farmland Environemntal Monitoring Applications," in IEEE 
Wireless Communications, Networking and Mobile Computing, 2009.

[10] G. G. Joshi, C. B. Dietrich Jr., C. R. Anderson, W. G. Newhall, W. A. Davis, J. Isaacs and G. Barnett, "Near-Ground Channel Measurements over Line-of-Sight and Forested Paths," IEEE Microwave, Antenna and Propagation Journal, vol. 152, no. 6, pp. 589-596, 2005.

[11] F. P. Correia, F. B. Carvalho, B. G. Leal and W. T. Lopes, "Propagation Analysis in Precision Agriculture Environment using XBee Devices," in Microwave \& Optoelecronics Conference (IMOC) IEEE MTTS-S International, Rio de Janeiro, 2013.

[12] A. Alsayyari, I. Kostanic, C. Otero, M. Almeer and K. Rukieh, "An Emprical Path Loss Model for Wireless Sensor Network Deployment in a Sand Terrain Environment," in Internet of Things (WF-IoT), 2014 IEEE World Forum, Seoul, 2014.

[13] A. AlSayyari, I. Kostanic and C. E. Otero, "An Empirical Path Loss Model Wireless Sensor Network Deployment in an Artificial Turf Evironment," in Networking, Sensing and Control (ICNSC), 2014 IEEE 11th International Conference, Maimi, FL, 2014.

[14] A. Alsayyari, I. Kostanic and C. E. Otero, "An Empirical Path Loss Model for Wireless Sensor Network Deployment in a Concrete Surface Environment," in Wireless and Microwave Technology Conference (WAMICON), 2015 IEEE, Cocoa Beach, 2015.

[15] D. Montegomery, Design and Analysis of Experiemnts, 8 ed., Wiley, John \& Son, 2012.

[16] M. Paavola and M. Ruusunen, "Some Factors Affecting Performance of a Wireless Snesor Network - Entropy-Based Analysis," in Emerging Tchnologies and Factory Automation, IEEE internatioal Conference, Hamburg, 2008.

[17] O. Chipara, G. Hackman, C. Lu, W. D. Smart and C. G. Roman, "Practical Modeling and Prediction of Radio Coverage of Indoor Sensor Nerworks," in IPSN, ACM, Sweden, 2010.

[18] P. Abouzar and D. G. Michelson, "Characterization of Wireless Mesh Network Performance in Agricultural Environment," in Radio Science Meeting, USN-URSI, IEEE, Memphis TN, 2014.

[19] M. Pisu, G. Morariu and F. Sandu, "RSSI-Based Electromagnetic Propagation in Industrial Environment," in RoEduNet IEEE Conference, Chisinau, 2014.

[20] S. Temel, N. Unaldi and O. Kaynak, "On Deployment of Wireless Sensors on 3-D Terrains to Maximize Sensing Coverage by Utilizing Cat Swarm Optimization With Wavelet Transform," IEEE Transactions on Systems, Man, and Cybernetics: Systems, vol. 44, no. 1, pp. 111 - 120, 2014.

[21] H. M. La, W. Sheng and J. Chen, "Cooperative and Active Sensing in Mobile Sensor Networks for Scalar Field Mapping," IEEE Transactions on Systems, Man, and Cybernetics: Systems, vol. 45, no. 1, pp. 1-12, 2015.

[22] P.-H. Tsai, Y.-J. Lin, Y.-Z. Ou, E. T.-H. Chu and J. W. S. Liu, "A Framework for Fusion of Human Sensor and Physical Sensor Data," IEEE Transactions on Systems, Man, and Cybernetics: Systems, vol. 44, no. 9, pp. 1248 - 1261, 2014.

[23] K. Ohshima, H. H. Hagiwara and M. Terada, "Field
Investigation of the Radio Transmission Perfornmance and Distance in a Environmental Wireless Sensor Network," in Information Networking (ICOIN), IEEE International Conference, Bali, 2012.

[24] H. Hongwei, S. Wei, X. Youzhi and Z. Hongke, "The Effect of Human Activities on $2.4 \mathrm{GHz}$ Radio Propagation at Home Environment," in Broadband Network \& Multimedia Technology (IC-BNMT) IEEE International Conference, Beijing, 2009.

[25] S. Hara, D. Zhao, K. Yanagihara, J. Taketsugu, K. Fukui, S. Fukunaga and K. Kitayama, "Propagation Characterization of IEEE 802.15.4 Radio Signal and Their Application for Location Estimation," in Vehicular Technology Conference (VTC), IEEE International Conference, 2005.

[26] W. T. Huang and H. D. Jeong, "A Novel High-Reliable Platform for Wireless Sensor Network with Serpentine-Antenna Design," in Complex, Inteligent and Software Intensive Systems, IEEE International Conference, Fukuoka, 2009.

[27] M. Wadhwa, M. Song, V. Rali and S. Shetty, "The Impact of Antenna Orientation on Wireless Sensor Network Performance," in Computer Science and Information Technology (ICCSIT), IEEE International Conference, Beijing, 2009.

[28] N. Yaakob, I. Khalil and H. Jiankun, "Performance Analysis of Optimal Packet Size for Congestion Control in Wireless Sensor Network," in Networking Computing and Applications (NCA), IEEE International Conference Symposium, Cambridge, MA, 2010.

[29] C. E. Otero, W. H. Shaw, I. Kostanic and L. D. Otero, "Mutliresonse Oprimization of Stochastic WSN Deployment Using Surface Methodology and Desirability Functions," IEEE Systems Journal Journal, vol. 4, no. 1, pp. 39-48, 2010.

[30] C. E. Otero, I. Kostanic and L. D. Otero, "A Decision-Making Methodology for Stochastic Deployment of Wireless Sensor Network," in Computer Modeling and Simulation (UKSIM), IEEE International Conference, Cambridge, 2009

[31] D. Montgonomery and G. Runger, Applied Statistics \& Probability for Engineers, 8 ed., John Wiley \& Son In, 2007.

[32] K. Kupper, N. Nizam and K. Muller, Applied Regression Analysis and other Multivariable Methods, 4th Edition ed., Brooks/Cengage Learning, 2008.

[33] "ZigBee Alliance," ZigBee Alliance, 2015. [Online]. Available: http://www.zigbee.org.

[34] D. P. Agrawal and Q.-A. Zeng, Introduction to Wireless and Mobile Systems, Cengage Learning, 2011.

[35] T. Olasupo, C. E. Otero, I. Kostanic and S. Shaikh, "Effects of Terrain Variations in Wireless Sensor Network Deployments," in IEEE International RF amd Microwave Conference, Sarawak, 2015.

[36] T. O. Olasupo, C. E. Otero, O. K. Olasupo and I. Kostanic, "Empirical Path Loss Models for Wireless Sensor Network Deployments in Short and Tall Natural Grass Environments," IEEE Transactions on Antennas \& Propagation, vol. 64, no. 9, pp. 4012-4021, 2016.

[37] T. O. Olasupo, C. E. Otero, K. O. Olasupo and A. Qureshi, "Automatic Detection of Radio Signal Obstruction in 
Wireless Sensor Networks' On-demand Deployments," in IEEE Sensor Symposium, Catania, Italy, 2016.

[38] L. Liu and H. Ma, "On Coverage of Wireless Sensor Networks for Rollings Terrains," Parallel and Distributd Systems, IEEE Transactions, vol. 23, no. 1, pp. 118-125, 2012.

[39] X. Bai, S. Kumar, D. Xuan, Z. Yun and T. Lai, "Deploying Wireless Sensors to Achieve Both Coverage and Connectivity," MobiHoc, ACM Journal Publication, 2006.
[40] L. Kong, M. Zhao, X. Liu, J. Lu, Y. Liu, M. Mu and W. Shu, "Surface Coverage in Sensor Networks," Parallel and Distributed Systems. IEEE Transactions, vol. 25, no. 1, pp. 234-243, 2014.

[41] Y. Kim, M. Yeo, D. Kim and K. Chung, "A Node Deployment Strategy Considering Environmental Factors and the Number of Nodes in Surveillance and Reconnaissance Sensor Networks," International Journal of Distributed Sensor Networks, 2012. 